\title{
Suppressive Effect of Huzhentongfeng on Experimental Gouty Arthritis: An In Vivo and In Vitro Study
}

\author{
Zi-cong Wu $\mathbb{D}^{1,2}$, Qiang Xue ${ }^{1},{ }^{3}$ Zhen-ling Zhao, ${ }^{2,4}$ Peng-jun Zhou, ${ }^{4}$ Qun Zhou, ${ }^{5}$ \\ Zhen Zhang, ${ }^{1,2}$ Jian-ping Deng, ${ }^{1,2}$ Ke Yang, ${ }^{2}$ Hua Fan, ${ }^{2}$ Yi-fei Wang, ${ }^{2,4}$ \\ and Zhi-ping Wang ${ }^{1}$ \\ ${ }^{1}$ Guangdong Provincial Engineering Center of Topical Precise Drug Delivery System, Department of Pharmaceutics, \\ Guangdong Pharmaceutical University, Guangzhou 510006, China \\ ${ }^{2}$ Guangzhou (Jinan) Biomedical Research and Development Center, Guangzhou 510632, China \\ ${ }^{3}$ Chenguang Biotech Group Co., Ltd., Handan 056000, China \\ ${ }^{4}$ College of Life Science and Technology, Jinan University, Guangzhou 510632, China \\ ${ }^{5}$ Huazhong University of Science and Technology, Wuhan 430074, China \\ Correspondence should be addressed to Zhi-ping Wang; wzping-jshb@gdpu.edu.cn \\ Zi-cong Wu and Qiang Xue contributed equally to this work.
}

Received 27 May 2019; Revised 24 August 2019; Accepted 17 September 2019; Published 4 December 2019

Guest Editor: Samuel Martins Silvestre

Copyright (c) $2019 \mathrm{Zi}$-cong Wu et al. This is an open access article distributed under the Creative Commons Attribution License, which permits unrestricted use, distribution, and reproduction in any medium, provided the original work is properly cited.

\begin{abstract}
Background. Huzhentongfeng (HZTF) is an extract from four Chinese medical herbs for treating gout. This study aims to evaluate its antigout activity and preliminary explore its mechanism in vivo and in vitro. Methods. The rats were intragastrically administered with HZTF for 5 days and then injected $0.1 \mathrm{ml}(10 \mathrm{mg})$ of MSU crystals to their joints for generating a gout model to analyze the paw volume and histopathology of joint synovial tissues of rats with different doses. We also investigated the antioxidant capacity of HZTF in vitro using indication including lipid peroxidation, $\mathrm{DPPH}$; and $\mathrm{ABTS}^{+}$radical-scavenging capacity; besides, we used qRT-PCR to measure the effect of HZTF on interleukin (IL)-1 $\beta$, caspase-1, NLRP3, and NQO1 expression in hydrogen peroxide-stimulated RAW264.7 macrophages and IL-1 $\beta$, IL-6, and tumor necrosis factor (TNF)- $\alpha$ in MSU crystal-induced THP- 1 monocytes. Confocal microscopy analysis was used to observe the dimerization of ASC adapter proteins. In addition, we also established quality standard of HZTF by using the high-performance liquid chromatography (HPLC) method. Results. HZTF could significantly suppress the paw swelling and neutrophil infiltration induced by MSU intra-articular injection in rats compared with the control group. HZTF also showed inhibition effects of inflammatory cytokines (IL-1 $\beta$, IL-6, and TNF- $\alpha$ ) secretion at 25.00 and $50.00 \mu \mathrm{g} / \mathrm{ml}$ in MSU-induced THP- 1 cells but showed no effects of IL-1 $\beta$, IL-6, and TNF- $\alpha$ mRNA expression in MSU-induced THP- 1 cells. Furthermore, confocal microscopy analysis showed that HZTF could prevent the oligomerization of ASC. Moreover, HZTF also showed effects in cell-free and cell-base tests of antioxidant capacity. Conclusion. The results prove that HZTF possessed the potential preventive effect against gout arthritis, and the effect may be attributed to its preventing effect on neutrophil infiltration and proinflammatory cytokines secretion such as IL-1 $\beta$, IL-6, and TNF- $\alpha$ which were caused by the activation of inflammasome.
\end{abstract}

\section{Introduction}

Gout is a common type of arthritis caused by hyperuricaemia and subsequent accumulation of monosodium urate crystal deposition in the joints, tendons, and surrounding tissues. The signs and symptoms of gout include sudden and intense pain, redness, swelling, and stiffness in a joint of the big toe or in another joint, such as the ankle, knee, elbow, wrist, and finger [1]. At present, there are many medications in clinic, such as nonsteroidal anti-inflammatory drugs (NSAIDs), colchicine, or corticosteroids, used to treat the symptoms of gout attacks, prevent future flares, and reduce the risk of gout complications. But they all have some limitations. Over $80 \%$ of patients treated with colchicine have experienced abdominal pain before full clinical improvement. In addition, the side effects of NSAIDs are more common in the elderly. 
Therefore, it is critical to develop more effective agents with less adverse reactions.

HZTF is extracted from four Chinese medical herbs which are widely used in China: Polygonum cuspidatum Sieb.et Zucc, Plantago depressa Willd, Ligustrum lucidum Ait, and Vespae nidus. These four medicinal materials have been used for treating gout for centuries [2-11].

The dried root of Polygonum cuspidatum Sieb.et Zucc was known as "Huzhang" in China, as a traditional Chinese medicine, which was officially listed in the Chinese Pharmacopoeia. The compounds from Plantago depressa Willd have been demonstrated to have tremendous amount of pharmacological activities, including antioxidant, antibacterial, and anti-inflammatory activities [12, 13]. Plantago depressa Willd contains large amounts of polyphenols such as resveratrol, and it is considered one of the best sources of resveratrol because it contains higher amounts of this compound than other plants or fruits [14]. Plantago depressa Willd or Cheqiancao in Chinese is widely distributed and utilised around world, which has no significant toxicities in a regular dosage and has been used as medicinal plants for centuries $[15,16]$. Currently, researches have shown that extracts from Plantain leaves exhibited antioxidant and antimicrobial activities. The above-mentioned bioactivities of Plantain leaves extracts are thought to be related to phenolic compounds and flavonoids [17]. Ligustrum lucidum Ait is a kind of evergreen shrub plant which can be found in Hu'nan, Jiangsu, Sichuan, and Zhejiang provinces in China. The mature fruits of this plant have a cardiac and roborant effect and known as a traditional herb medicine in China, which is also prescribed in Chinese Pharmacopoeia. Previous phytochemical investigations on Ligustrum lucidum Ait reported the isolation of secoiridoid glycosides, phenolic glycosides, and lignans $[18,19]$. Beehive is the hive of Parapolybia varia Fabricius, a widely used traditional Chinese medicine. It is widely distributed in China and be harvested in autumn and winter. After removing the dead wasps, the hive is manufactured into a kind of traditional Chinese medicine by openair drying. Because of its multiple pharmacological activities, including antivirus and anti-inflammatory beehive has been used in traditional Chinese medicine over thousands of years for treating various diseases, including malignant tumors, rheumatoid arthritis, lung diseases, skin disease, digestive and urinary disorders, and dental diseases $[11,20]$.

Huzhentongfeng (HZTF) is an extract developed by GuangZhou (Jinan) Biomedical Research and Development Center for treating gout. In order to obtain a good antigout effect, we combined the traditional Chinese medicine theory system with modern medical theory to extract these four medicinal materials to produce HZTF. According to the theory of traditional Chinese Medicine, Polygonum Cuspidatum is a monarch drug, Plantain is a minister drug, Ligustrum is an assistant drug, and Nidus Vespae is the guide drug. And, its main function is to treat acute gouty arthritis.

Firstly, in order to ensure the stability of product quality of HZTF, we also established a standard for the content of medicinal materials by using the HPLC method.

Synovial cells, monocytes-macrophages, and neutrophils will produce multiple cytokines, such as IL-1 $\beta$, IL-6, and
TNF- $\alpha$, when they were stimulated by MSU crystals in the joints. Our study demonstrated that HZTF showed prominent effect on neutrophil infiltration and paw swelling in rats induced by intra-articular MSU injection. However, the concrete mechanism of HZTF preventing gouty arthritis remains unclear. So, in this study, we investigated the inhibition effects on expression levels of IL- $1 \beta$, caspase-1, NLRP3, and NQO1 in hydrogen peroxide $\left(\mathrm{H}_{2} \mathrm{O}_{2}\right)$-induced RAW264.7 macrophages and expression and secretion of IL- $1 \beta$, IL- 6 , and TNF- $\alpha$ from MSU crystal-induced THP-1 cells to investigate the mechanism of HZTF in treating gouty arthritis.

Moreover, to investigate whether the antigout effect of HZTF was induced by its antioxidation activity, we tested the antioxidant effects of HZTF using lipid peroxidation, 1,1-diphenyl-2-picryl-hydrazyl free radical (DPPH)-scavenging activity, and 3-ethylbenzthiazoline-6-sulfonic acid (ABTS) radical-scavenging activity in vitro.

\section{Materials and Methods}

2.1. Equipment. Stainless steel tape gauge, produced by Southwest China Tool General Plant of Guizhou Aviation Industry Group, China. The model of UV-visible spectrophotometer used in this experiment is UV2600 (Shimadzu, Japan), the model of the centrifugal machine is HC-2518 (Anhui USTC Zonkia, China), the real-time thermal cycler is CFX96 Touch (BioRad, USA), and the microplate reader is ELx808 (BioTek, USA).

2.2. Reagents. HZTF was produced by Yili Pharmaceutical Co., Ltd., Guangdong, China. Tongfengshu was produced by Qinghai Green Pharmaceutical Co., Ltd, Qinghai, China. Dexamethasone (DEX) and MCC950 were produced by Selleck, Shanghai, China. Phorbol-12-myristate-13-acetate (PMA), ascorbic acid (VC), ABTS, DPPH, AAPH, tris, ferrozine, ammonium thiocyanate, methanol, ethyl alcohol, hydrochloric acid, potassium peroxydisulfate, ferric chloride, sodium dihydrogen phosphate, disodium hydrogen phosphate, sodium phosphate, hydrogen peroxide, uric acid, sodium hydroxide, MTT, and potassium persulfate were all in analytical grade and obtained from Aladdin Industrial Corporation, Shanghai, China. PCR Mastermix and cDNA reverse transcriptase kit were obtained from Takara, Beijing China. TRIzol reagent was from Tiangen, Beijing, China. Gene specific primers were from Sangon, Shanghai, China. Elisa kits were purchased from neobioscience, Guangdong, China. Anti-ASC antibody was from Proteintech, Illinois, USA.

2.3. Quality Standard of HZTF by HPLC. Quality standard of HZTF was performed as described in an earlier report with slight modifications [21]. Briefly, HZTF was dissolved in $70 \%$ ethanol, and HPLC analysis was conducted using Poroshell 120 SB-C18 $(4.6 \times 100 \mathrm{~mm}, 2.7 \mu \mathrm{m}$, Agilent, US). The chromatogram was monitored at wavelength $280 \mathrm{~nm}$. The mobile phase consisted of A ( $0.1 \%$ formic acid) and $\mathrm{B}$ (acetonitrile) with an isometric elution as follows: A 16\% and 
B $84 \%$. The flow rate of the mobile phase was $1.0 \mathrm{ml} / \mathrm{min}$, and the injection volume was $10 \mu \mathrm{l}$.

2.4. Synthesis of MSU Crystals. MSU crystals were synthesized as described [22]. Briefly, $1.68 \mathrm{~g}$ of uric acid in $0.01 \mathrm{M}$ $\mathrm{NaOH}$ was in a water bath heated to $70^{\circ} \mathrm{C}$. Then, $0.5 \mathrm{M}$ $\mathrm{NaOH}$ was added to maintain $\mathrm{pH}$ between 7.1 and 7.2, and the solution was incubated at room temperature with slow stirring and continuously for $24 \mathrm{~h}$. The crystals were washed with absolute ethyl alcohol, freeze dried, and autoclaved.

2.5. Animals. Male Sprague-Dawley rats $(200 \pm 20 \mathrm{~g})$ were purchased from the Experimental Animal Center of Henan Province, China. The animals were allowed to adapt to their environment for at least one week before initiation of the experiments. The animals were housed five per cage under a normal $12 \mathrm{~h} / 12 \mathrm{~h}$ light/dark schedule. The animals were housed at room temperature $\left(20 \pm 2^{\circ} \mathrm{C}\right)$ with relative humidity $(60 \pm 5 \%)$ and were free access to a standard diet in the duration of the study. All studies were operated in accordance with the Institutional Animal Care Committee at the Experimental Animal Center of Henan Province and the China Council on Animal Care at the Experimental Animal Center of Henan Province.

\subsection{MSU Crystal-Induced Inflammation in Rats}

2.6.1. Animal Gout Model and Drug Administration. The rats were randomly divided into six groups. Each group contained ten rats. Group I served as controls and were intra-articular injected in the right posterior foot with $0.1 \mathrm{ml}$ physiological saline. In group II, gouty inflammation was induced by intra-articular injection of $0.1 \mathrm{ml}(10 \mathrm{mg})$ of MSU crystal suspension into the right posterior foot. Groups III, IV, and V comprised MSU crystal-induced rats treated with $\operatorname{HZTF}(0.25,0.50$, and $1.00 \mathrm{~g} / \mathrm{kg}$ body weight, respectively). Group VI comprised MSU crystal-injected rats treated with positive control Tongfengshu (a commercially available Chinese patent medicine for gout, $1.50 \mathrm{~g} / \mathrm{kg}$ body weight). HZTF and Tongfengshu were fully dispersed in distilled water. All doses of the respective drugs were expressed as grams per kilogram body weight. Drugs were intragastrically administered for $5 \mathrm{~d}$ once daily and also administration at the 5 th day $1 \mathrm{~h}$ before the MSU crystal injection. Isoflurane was used to anesthetize the rats, and then the MSU suspension injection was given.

2.6.2. Assessment of Paw Swelling. The pathological degree was quantified by the swelling value of paw measured at 1,2 , $3,4,5,6$, and $7 \mathrm{~h}$ after MSU crystal injections. The degree of edema was expressed as paw swelling which was calculated by the following equation:

$$
\text { foot swelling value }=b-a \text {. }
$$

Here, $a$ is the foot volume before MSU crystal injection and $b$ is the foot volume after MSU crystal injection.
2.6.3. Histopathological Analysis. Rats were killed by cervical dislocation $(7 \mathrm{~h})$, and then the ankle joints of the right posterior foot of the rats were dissected and fixed in $10 \%$ neutral formaldehyde fixative for synovial tissue collection. After decalcification, the tissues were paraffin-embedded, sectioned, and stained with haematoxylin and eosin (H\&E).

\subsection{Cell-Free In Vitro Studies}

2.7.1. Lipid Peroxidation. The thiobarbituric acid (TBA) method was carried out according to the method described by Costa et al. [23-25], and modified according to our situation. Briefly, $1 \mathrm{~mL}$ of the different concentrations of HZTF or VC was mixed with $1 \mathrm{~mL}$ of $10 \%$ yolk homogenate in tris buffer ( $\mathrm{pH} 8.0$ ). Then, $100 \mu \mathrm{l}$ of $70 \mathrm{mM}$ AAPH, $2.9 \mathrm{ml}$ $20 \%$ acetic acid, and $3.0 \mathrm{ml} 0.8 \%$ TBA solution were added. And, the mixture was boiled 60 min for full reaction; after cooling, adding $4.0 \mathrm{ml}$ butanol, and fully extracting, the mixture was centrifuged at $2000 \mathrm{~g}$ for $10 \mathrm{~min}$, extracting the organic layer. The final concentrations of HZTF or VC in the reaction system are 30.00, 60.00, 90.00, 120.00, and $150.00 \mu \mathrm{g} / \mathrm{ml}$. The absorbance was measured at $532 \mathrm{~nm}$ by the ultraviolet-visible spectrophotometer. The decrease of absorbance indicates the increase of antioxidant activity of the samples. The antioxidant activity value of the samples was expressed as the percentage of lipid peroxidation inhibition rate which was calculated by the following equation:

$$
\text { inhibition effect }(\%)=\left(1-\frac{A_{\mathrm{S}}}{A_{\mathrm{C}}}\right) \times 100 \text {, }
$$

where $A_{\mathrm{S}}$ is the absorbance of test sample and $A_{\mathrm{C}}$ is the absorbance of blank control.

2.7.2. DPPH Radical-Scavenging Ability. DPPH radicalscavenging capacity was determined according to the modified method by Luo et al. [26]. HZTF and VC samples were prepared in $70 \%$ ethanol at the difference concentrations, then $1 \mathrm{ml}$ of each sample mixed with $1 \mathrm{ml} \mathrm{DPPH}$ solution ( $0.1 \mathrm{M}$ in $50 \%$ ethanol) and $3 \mathrm{ml}$ distilled water. The final concentrations of HZTF are 4.00, 12.00, 20.00, 28.00 and $36.00 \mu \mathrm{g} / \mathrm{ml}$, and $\mathrm{VC}$ are $2.00,4.00,6.00,8.00$ and $10.00 \mu \mathrm{g} / \mathrm{ml}$. The mixtures were vortexed and allowed to equilibrate for $20 \mathrm{~min}$ at room temperature in the dark, and then measured the absorbance at $517 \mathrm{~nm}$. [27, 28]. Scavenging activity was calculated using the formula:

$$
\text { scavenging effect }(\%)=\left(1-\frac{A_{\mathrm{S}}}{A_{\mathrm{C}}}\right) \times 100 \text {, }
$$

where $A_{\mathrm{S}}$ is the absorbance of the samples at different concentration and $A_{\mathrm{C}}$ is the absorbance of control.

2.7.3. ABTS $^{+}$Radical-Scavenging Capacity. ABTS radical cation $\left(\mathrm{ABTS}^{+}\right.$radical)-scavenging capacity was carried out according to the method of $\mathrm{Re}$ et al. with modification according to the situation in our laboratory [29-31]. ABTS stock solution $(3.0 \mathrm{mg} / \mathrm{ml})$ was prepared by dissolving ABTS in $2.5 \mathrm{mM}$ potassium persulfate solution and incubated the 
mixture in dark overnight at room temperature. The ABTS solution was diluted with sodium phosphate buffer $(\mathrm{pH} 7.4)$ to obtain absorbance of $0.7 \pm 0.1$ at $734 \mathrm{~nm} .4 \mathrm{ml}$ of HZTF or $\mathrm{VC}$ in different concentrations were mixed with $4 \mathrm{ml}$ of $\mathrm{ABTS}^{+}$radical working solution and reacted for $300 \mathrm{~s}$. Then, the absorbance was measured at $734 \mathrm{~nm}$. The final concentrations of HZTF were 5.00, 7.50, 10.00, 12.50, and $15.00 \mu \mathrm{g} /$ $\mathrm{ml}$, and VC were $1.00,2.00,3.00,4.00$, and $5.00 \mu \mathrm{g} / \mathrm{ml}$.

$$
\mathrm{ABTS}^{+} \text {radical scavenging }(\%)=\left(1-\frac{A_{\mathrm{S}}}{A_{\mathrm{C}}}\right) \times 100 \text {, }
$$

where $A_{\mathrm{S}}$ is the absorbance of sample and $A_{\mathrm{C}}$ is the absorbance of the black control.

\subsection{Cell-Base In Vitro Studies}

2.8.1. Cell Culture. RAW murine macrophage 264.7 (RAW264.7) and THP-1 cell lines were purchased from the Institute of Biochemistry and Cell Biology, CAS, Shanghai, China. The RAW264.7 were cultured in Dulbecco's modified eagle's medium (DMEM) with 10\% fetal bovine serum (FBS), 1x antibiotic solution (streptomycin $(100 \mathrm{U} / \mathrm{ml})$ and penicillin $(100 \mathrm{U} / \mathrm{ml}))$ in a humidified atmosphere supplied with $5 \% \mathrm{CO}_{2}$ and maintained at a temperature of $37^{\circ} \mathrm{C}$. Cells were allowed to grow till they reached a confluency of $80-90 \%$ and washed with phosphate buffered saline (PBS) with regular replacement of culture medium.

THP-1 cells were maintained in 1640 medium supplemented with $10 \%$ FBS in a humidified atmosphere supplied with $5 \% \mathrm{CO}_{2}$ and maintained at temperature of $37^{\circ} \mathrm{C}$. Cells were allowed to grow till they reached a density of $2 \times 10^{6}$ and then added the same volume of culture medium.

2.8.2. Cell Viability. Cell viability was performed by MTT assay. RAW264.7 macrophage cells were seeded in a 96-well culture plate. THP-1 cells were seeded in another 96-well culture plate for 3 hours with $30.82 \mu \mathrm{g} / \mathrm{ml}$ PMA, the day before drug treatment. After $24 \mathrm{~h}$ incubation, the cells were stimulated with varying concentrations of $\operatorname{HZTF}(3.12,6.25$, $12.50,25.00,50.00,100.00$, and $200.00 \mu \mathrm{g} / \mathrm{ml}$ ) in FBS-free medium for further $24 \mathrm{~h}$. After incubation, $10.0 \mu \mathrm{L}$ MTT DMSO solutions ( $5 \mathrm{mg} / \mathrm{ml}$ ) were added to each well. Then, the plates were incubated for $4 \mathrm{~h}$ at $37^{\circ} \mathrm{C}$ in dark. After incubation, $90 \mu \mathrm{L}$ medium was removed from each well, $100 \mu \mathrm{L}$ of DMSO was added to the wells, and then the plates were incubated for $15 \mathrm{~min}$ at $37^{\circ} \mathrm{C}$ under gentle shaking to dissolve the tetrazolium dye. The cell viability was calculated according to the results of absorbance at $570 \mathrm{~nm}$ and expressed as relative percentage viability. The relative cell percentage viability was calculated by the following equation:

$$
\text { cell viability }(\%)=\left[\frac{A_{\mathrm{S}}-A_{\mathrm{b}}}{A_{\mathrm{C}}-A_{\mathrm{b}}}\right] \times 100 \text {, }
$$

where $A_{\mathrm{S}}$ is the absorbance of HZTF, $A_{\mathrm{C}}$ is the absorbance of the control, and $A_{\mathrm{b}}$ is the absorbance of cell blank.
2.8.3. Cell Treatment. Cells reaching a concentration of $5 \times 10^{6}$ cells/well in 12-well culture plate were considered for drug treatment. RAW264.7 macrophages were treated with varying concentrations of HZTF $(100.00$ and $200.00 \mu \mathrm{g} / \mathrm{ml})$ and $\mathrm{H}_{2} \mathrm{O}_{2}(882.09 \mathrm{nM})$ for $24 \mathrm{~h}$ and prepared HZTF DMSO working solution in different concentrations. THP-1 cells were seeded with $30.82 \mu \mathrm{g} / \mathrm{ml}$ PMA in a 24-well culture plate for 3 hours the day before drug treatment. After $24 \mathrm{~h}$ of incubation, the cells were stimulated with varying concentrations of $\operatorname{HZTF}(6.25,12.50,25.00$ and $50.00 \mu \mathrm{g} / \mathrm{ml})$ and MSU in FBS-free medium for further $8 \mathrm{~h}$, and then the cell culture medium of each well was collected for Elisa analysis.

2.8.4. Quantification of $m R N A$ Levels by Quantitative RealTime PCR. Total RNA was isolated from RAW264.7 macrophages treated with various concentrations of DEX $(25 \mu \mathrm{g} /$ $\mathrm{ml})$ and HZTF $(100.00$ and $200.00 \mu \mathrm{g} / \mathrm{ml})$ with $\mathrm{H}_{2} \mathrm{O}_{2}(0.15 \%)$ stimulation for $24 \mathrm{~h}$ and THP-1 cells pretreated with HZTF $(25.00$ and $50.00 \mu \mathrm{g} / \mathrm{ml})$ and stimulated by MSU $(1 \mathrm{mg} / \mathrm{ml})$ for $8 \mathrm{~h}$ using the TRIzol reagent according to manufacturer's instructions. The total RNA was reverse transcribed by using cDNA reverse transcriptase kit, and then mRNA expression was amplified by PCR mastermix. Gene specific primers were designed manually using online NCBI primer-BLAST tool. Primer sequences of forward and reverse are shown in Table 1. Quantitative real-time PCR (qRT-PCR) was used to analyse the IL- $1 \beta$, caspase-1, NLRP3, NQO1, and GAPDH gene expression of RAW 264.7 and IL-1 $\beta$ of THP-1, respectively. Transcription levels were assessed to utilize the step on real-time thermal cycler with PCR mastermix. Thermal cycling conditions were as follows: denaturation at $95^{\circ} \mathrm{C}$ for $5 \mathrm{~s}$, annealing at $60^{\circ} \mathrm{C}$ for $30 \mathrm{~s}$, and extension at $65^{\circ} \mathrm{C}$ for $5 \mathrm{~s}$. The levels of GAPDH gene expression served as an internal control.

2.8.5. ELISA Analysis. THP-1 cells were pretreated with MCC950 $(4.26 \mu \mathrm{g} / \mathrm{ml})$ or HZTF $(6.25,12.50,25.00$ and $50.00 \mu \mathrm{g} / \mathrm{ml})$, stimulated by MSU $(1 \mathrm{mg} / \mathrm{ml})$ for $8 \mathrm{~h}$, and collected the supernatant. Secretion of inflammatory cytokine (IL- $1 \beta$, IL- 6 and TNF- $\alpha$ ) was measured by ELISA kits according to the assay procedure.

2.8.6. Western Blotting Analysis. Protein samples were obtained from the culture medium as same as in the ELISA assay and concentrated by the ultrafiltration device. Protein sample was denatured by mixing with $1 / 4$ volume of $5 \mathrm{x}$ loading buffer, and metal bath at $100^{\circ} \mathrm{C}$ for $10 \mathrm{~min}$. Samples were resolved by electrophoresis with 10\% SDS-PAGE and transferred onto a PVDF membrane (Merk, Germany). After blocking nonspecific binding sites for $2 \mathrm{~h}$ with $5 \%$ dried skim milk dissolved in TBST, the membranes were individually incubated for overnight with anti-IL- $1 \beta$, antiIL-6, anti-caspase-1, anti- PGE2, and ASC. Develop the color of the blot rocking in 3, $3^{\prime}$-diaminobenzidine (DAB) substrate solution. Stop the reaction by pouring out the substrate after the expected band appears and then well rinsed 
TABLE 1: Sequences of PCR primers used for qRT-PCR.

\begin{tabular}{|c|c|c|c|}
\hline Species & Gene & Primer & Sequence \\
\hline \multirow{10}{*}{ Mice } & \multirow{2}{*}{ IL- $1 \beta$} & Forward primer & ACCTGGGCTGTCCTGATGAGAG \\
\hline & & Reverse primer & TGTTGATGTGCTGCTGCGAGAT \\
\hline & \multirow{2}{*}{ Caspase-1 } & Forward primer & GGGACCCTCAAGTTTTGCC \\
\hline & & Reverse primer & CAACTTGAGCTCCAACCСТC \\
\hline & \multirow{2}{*}{ NLRP3 } & Forward primer & TCTGTGTGGACCTAAGCCCC \\
\hline & & Reverse primer & GGGATACAGCCTTTCTCGGG \\
\hline & \multirow{2}{*}{ NQO1 } & Forward primer & GAGAGGATGGGAGGTACTC \\
\hline & & Reverse primer & AATATCTGGGCTCAGGCGTC \\
\hline & \multirow{2}{*}{ GAPDH } & Forward primer & GTCATTGAGAGCAATGCCAG \\
\hline & & Reverse primer & GTGTTCCTACCCCCAATGTG \\
\hline \multirow{8}{*}{ Human } & \multirow{2}{*}{ IL- $1 \beta$} & Forward primer & TGATGGCTTATTACAGTGGCAATG \\
\hline & & Reverse primer & GTAGTGGTGGTCGGAGATTCG \\
\hline & \multirow{2}{*}{ IL-6 } & Forward primer & CACTGGTCTTTTGGAGTTTGAG \\
\hline & & Reverse primer & GGACTTTTGTACTCATCTGCAC \\
\hline & \multirow{2}{*}{ TNF- $\alpha$} & Forward primer & CAGAGGGAAGAGTTCCCCAG \\
\hline & & Reverse primer & CCTTGGTCTGGTAGGAGACG \\
\hline & \multirow{2}{*}{ GAPDH } & Forward primer & AGCCTCAAGATCAGCAATG \\
\hline & & Reverse primer & CACGATACCAAAGTTGTCATGGAT \\
\hline
\end{tabular}

with distilled water repeatedly. Dry the membrane, and store it in the dark place.

2.8.7. Confocal Microscopic Analysis. THP-1 cells were pretreated with MCC950 $(4.26 \mu \mathrm{g} / \mathrm{ml})$ or HZTF $(50.00 \mu \mathrm{g} /$ $\mathrm{ml}$ ) and stimulated by MSU ( $1 \mathrm{mg} / \mathrm{ml})$ for $8 \mathrm{~h}$. Briefly, THP-1 cells were plated on coverslips and incubated with an antiASC antibody and then incubated with an anti-rabbit IgGFITC antibody. Recording with a laser scanning confocal microscope (LSM710, Carl Zeiss, Oberkochen, Germany), the ASC speck formation was analyzed using Zen2010 software.

2.9. Statistical Analysis. All results were expressed as mean \pm standard deviation $(n=3)$. Statistical analysis was carried out by SPSS 21 statistical software (IBM Inc., USA). A statistical difference was considered significant when $P$-values are less than 0.05 .

\section{Results}

3.1. Quality Standard of HZTF by HPLC. As shown in Figure 1, four substances in HZTF were selected as content indicators: (1) plantamajoside, (2) polygonum cuspidin, (3) specnuezhenide, and (4) resveratrol glucoside, and all of them had distinct peaks under $280 \mathrm{~nm}$ ultraviolet absorption.

\subsection{Effect of HZTF and Tongfengshu in In Vivo Studies.} To determine the degree of edema, the swelling value of the right posterior foot ankle joints of rats were measured (Table 2). The foot-swelling values of MSU crystal-induced rats were significantly higher than that of the control rats at $1 \mathrm{~h}$ to $7 \mathrm{~h}(P<0.05)$. Paw edema decreased significantly in MSU crystal-induced rats treated with HZTF (0.50 and $1.00 \mathrm{~g} / \mathrm{kg}$ body weight) at $1 \mathrm{~h}$ to $7 \mathrm{~h}$ and Tongfengshu $(1.50 \mathrm{~g} /$ kg body weight) at $1 \mathrm{~h}$ to $7 \mathrm{~h}(P<0.05)$.
To compare the histological changes in the joints between different groups, joint tissues were sectioned and examined via $H \& E$ staining. The coverage of synovial cells on synovial cavity of the joints was fragmentary, and there was edema in loose connective tissue, as well as local infiltration of leukocytes (Figure 2). However, rats treated with HZTF (0.50 and $1.00 \mathrm{~g} / \mathrm{kg}$ body weight) and Tongfengshu $(1.50 \mathrm{~g} / \mathrm{kg}$ body weight) decreased edema and leukocyte infiltration after $7 \mathrm{~h}$. This change correlated well with the decrease in paw swelling induced by HZTF and Tongfengshu treatment.

3.3. In Vitro Cell-Free Antioxidation Experiment of HZTF. To investigate whether the antigout effect of HZTF is due to its antioxidant activity, we measured the antioxidant activity of HZTF using multiple methods. As shown in Figure 3(a), we can know that HZTF can significantly inhibit lipid peroxidation when the concentration was higher than $60.00 \mu \mathrm{g} / \mathrm{ml}$, and the $\mathrm{IC}_{50}$ values of $\mathrm{VC}$ and HZTF are $49.94 \pm 3.67$ and $114.62 \pm 3.43 \mu \mathrm{g} / \mathrm{ml}$, respectively, which mean the lipid peroxidation inhibition ability of HZTF is significantly weaker than VC $(P<0.05)$. It is observed that the $\mathrm{DPPH}^{-}$radical-scavenging activity of HZTF is shown in Figure $3(\mathrm{~b})$. The $\mathrm{IC}_{50}$ values of VC and HZTF are $2.81 \pm 0.62$ and $13.04 \pm 2.80 \mu \mathrm{g} / \mathrm{ml}$, respectively. Though it can inhibit the $\mathrm{DPPH}^{-}$radical, the $\mathrm{IC}_{50}$ value of HZTF is significantly higher than $\mathrm{VC}(P<0.05)$. As shown in Figure $3(\mathrm{c})$, the $\mathrm{ABTS}^{+}$radical-scavenging activity of both VC and HZTF are in dose-effect relationships. HZTF can scavenge the $\mathrm{ABTS}^{+}$ radical significantly when the concentration was higher than $5.00 \mu \mathrm{g} / \mathrm{ml}$ and the $\mathrm{IC}_{50}$ values of $\mathrm{VC}$ and HZTF are $1.48 \pm 0.70$ and $7.52 \pm 0.50 \mu \mathrm{g} / \mathrm{ml}$, respectively, which means the $\mathrm{ABTS}^{+}$radical-scavenging activity of HZTF is significantly weaker than VC $(P<0.05)$.

3.4. Cell Viability. MTT assay was utilized to measure the cytotoxicity of HZTF on RAW264.7 macrophages and THP- 


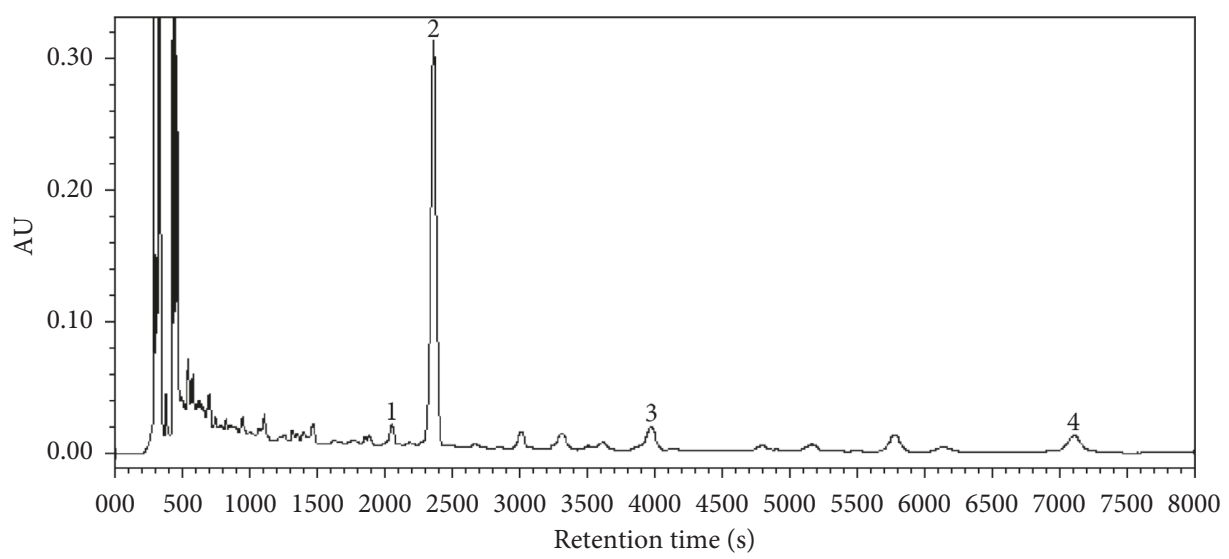

FIGURE 1: The quality standard of HZTF was determined using an HPLC system, plantamajoside (1), polygonum cuspidin (2), specnuezhenide (3) and resveratrol glucoside (4).

1 cells. Figure 4 shows that the cell viability was not significantly altered after treating with HZTF for $24 \mathrm{~h}$ up to $200.00 \mu \mathrm{g} / \mathrm{ml}$ concentration, suggesting no cytotoxicity of HZTF up to $200.00 \mu \mathrm{g} / \mathrm{ml}$.

\subsection{Experiment with RAW264.7 Macrophages}

3.5.1. RT-qPCR Analysis. As shown in Figure 5, changes of IL-1 $\beta$, caspase-1, NLRP3, and NQO1 expression in $\mathrm{H}_{2} \mathrm{O}_{2-}$ induced RAW264.7 macrophages were compared with the control group without $\mathrm{H}_{2} \mathrm{O}_{2}$, and the expression of IL- $1 \beta$, caspase-1, and NLRP3 in RAW264.7 macrophages was increased by treatment with $0.15 \% \mathrm{H}_{2} \mathrm{O}_{2}$ for $24 \mathrm{~h}$. Interestingly, the expression of IL- $1 \beta$, caspase- 1 , and NLRP3 in RAW264.7 macrophages treated with HZTF at 100.00 and $200.00 \mu \mathrm{g} / \mathrm{ml}$ were significantly reduced $(P<0.05)$. Moreover, an obvious difference $(P<0.05)$ was found between the mRNA expression of NQO1 with or without HZTF (100.00 and $200.00 \mu \mathrm{g} / \mathrm{ml}$ ), and the expression of NQO1 was increased with the increase of HZTF concentrations, which may indicate a dose-dependent relationship between NQO1 expression and HZTF concentrations. In conclusion, the results showed that $\mathrm{H}_{2} \mathrm{O}_{2}$ upregulated the expression of IL$1 \beta$, caspase- 1 , and NLRP3 and downregulated the expression of NQO1. Treatment with HZTF inhibits the expression of IL- $1 \beta$, caspase-1, and NLRP3 and enhanced the expression of NQO1 in $\mathrm{H}_{2} \mathrm{O}_{2}$-induced RAW264.7 macrophages at the same time.

\subsection{Experiment with THP-1 Cells}

3.6.1. ELISA Analysis. To investigate the mechanism of HZTF against gout, qPCR was used to measure the effect of HZTF on IL- $1 \beta$, IL- 6 , and TNF- $\alpha$ expression and ELISA was used to measure the effect of HZTF on IL- $1 \beta$, IL- 6 , and TNF$\alpha$ secretion. As shown in Figures 6(a)-6(c) MSU or HZTF does not significantly affect the expression of IL-1 $\beta$, IL-6, and TNF- $\alpha$. As shown in Figures 6(d)-6(f), HZTF significantly reduced the secretion of IL- $1 \beta$, IL- 6 , and TNF- $\alpha$ in THP- 1 cells stimulated by MSU $(P<0.05)$. The IC 50 of IL- $1 \beta$, IL-6, and TNF- $\alpha$ secretion in THP-1 cells stimulated by
MSU were $20.94 \pm 2.13,26.04 \pm 1.28$, and $24.87 \pm 0.68 \mu \mathrm{g} / \mathrm{ml}$, respectively.

3.7. Western Blotting Analysis. As shown in Figure $7 \mathrm{HZTF}$ inhibited the procaspase-1, cleaved-caspase-1, pro-IL- $1 \beta$, cleaved-IL- $1 \beta$, ASC dimer, IL-6, and PGEs 2 in the culture medium which were secreted by THP-1 cells in a dosedependent manner.

3.8. Confocal Microscopic Analysis. As a symbol of inflammasome activation, ASC forms oligomers in response to NLRP3 activators. Confocal microscopy analysis intuitively showed that HZTF suppressed MSU crystal-induced formation of ASC speckles in THP-1 cells (Figure 8).

\section{Discussion}

Inflammatory response to MSU crystals triggered the acute symptoms of gout and mainly mediated by macrophages and neutrophils. Intra-articular injection of MSU crystal can simulate gouty arthritis in humans and produces a painful response which is similar to the acute gouty attacks occurrence spontaneously [32]. Significant swelling accompanied by an extensive inflammatory response after MSU injection in the ankle joint is an important symbol in determining the degree of inflammation and therapeutic efficacy. The primary pathological symbol of gout is the neutrophils accumulated and infiltrated in the joint tissues, which actively phagocytosed MSU crystals. Then, the membrane lysed and released inflammatory cytokines and free radicals amplified the inflammatory response [33, 34]. We observed similar changes in the joint MSU injection rat model, so this model is suitable for studying the pathological mechanism of gout arthritis.

Plants which give antioxidant activities should be highly potent in the management of gout because they often share xanthine oxidase (XO) inhibitory effects [35, 36]. We measured the antioxidant activities to study whether the antigout effect of HZTF is related to its antioxidant capacity. Lipid peroxidation occurs in the process of reactive oxygen 


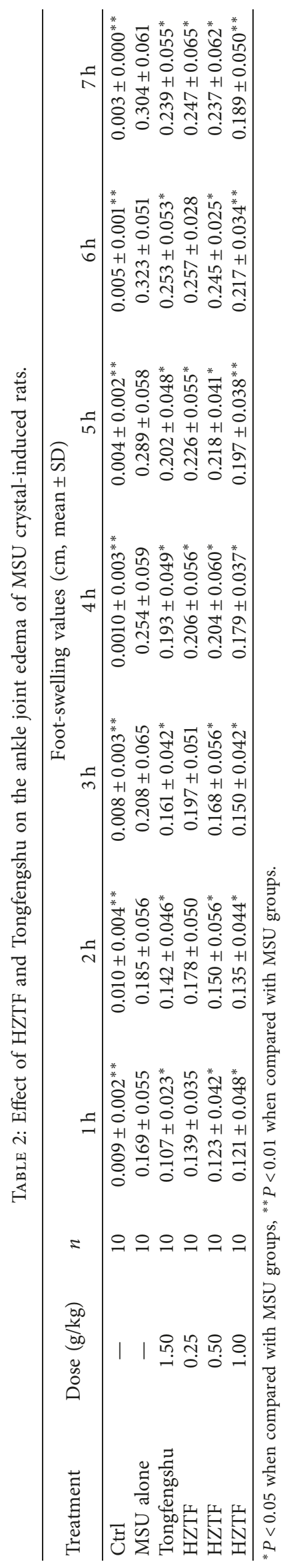




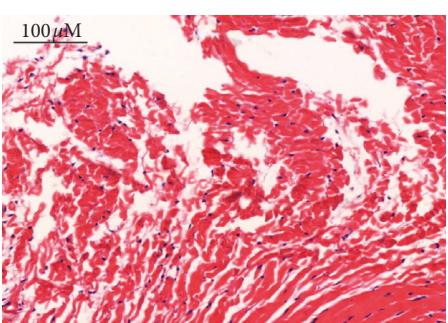

(a)

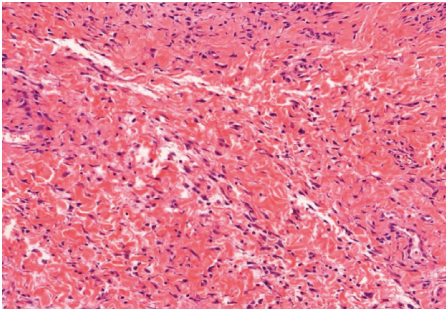

(c)

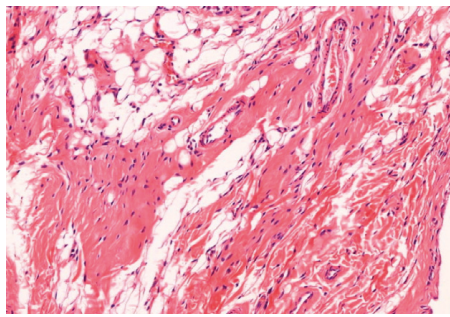

(e)

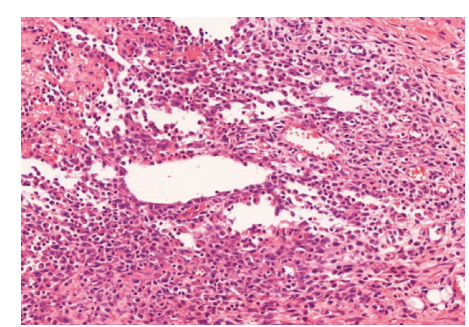

(b)

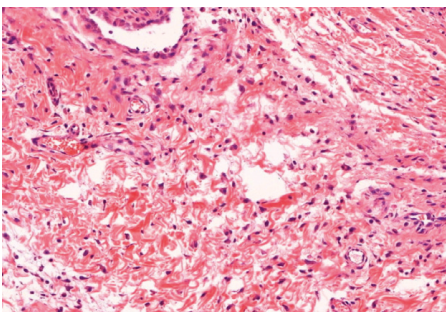

(d)

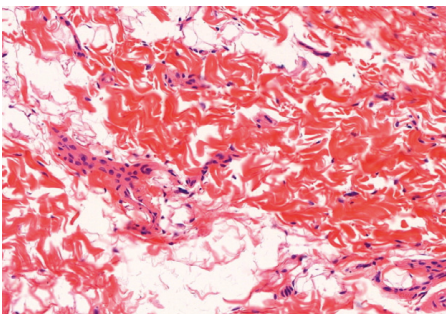

(f)

FIGURE 2: Histological examination of rat joints injected with MSU crystals (magnification, $\times 200$ ). In the control group, synovial membrane was normal and no inflammatory cell infiltration was found. (a) Joints injected with MSU crystals demonstrated leukocytes infiltrations and edema. Synovial tissue proliferation of nodularity was observed in the synovial lining of the joint, as well as synovial membrane cells with some zones of degeneration and necrosis (MSU alone). (b) Acute inflammation with polymorphonuclear infiltration in the synovial lining of MSU crystal-injected rats was attenuated by Tongfengshu and HZTF. (c) The Tongfengshu $(1.50 \mathrm{~g} / \mathrm{kg})$ group has no obvious edema in loose connective tissue, but there were still demonstrated inflammatory cells infiltration. (d-f) HZTF $(0.25,0.5$ and $1.00 \mathrm{~g} / \mathrm{kg}) \mathrm{groups}$ demonstrated only mild infiltration of scattered leukocytes. The synovial of HZTF group (MSU and HZTF $1.00 \mathrm{~g} / \mathrm{kg}$ ) appeared normal.

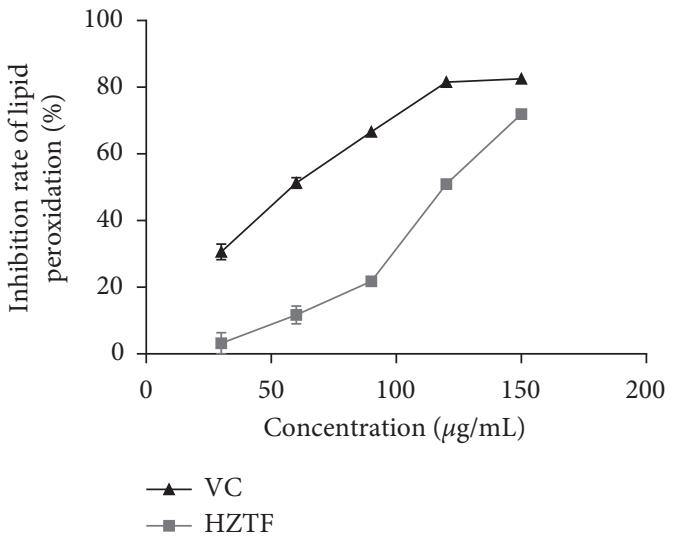

(a)

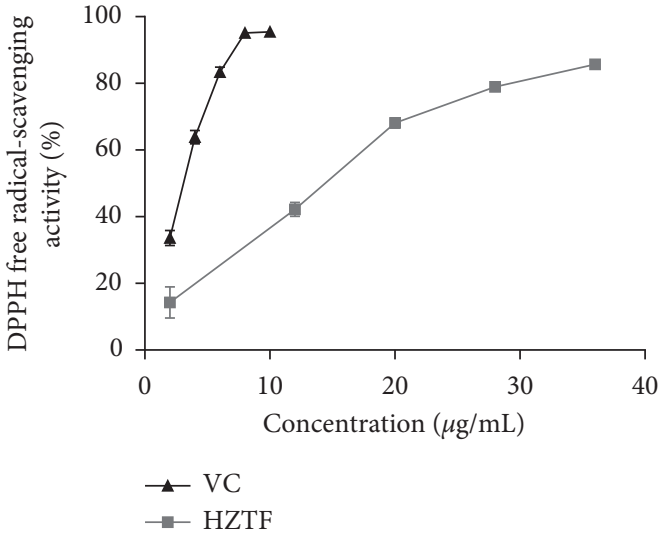

(b)

FIgURE 3: Continued. 


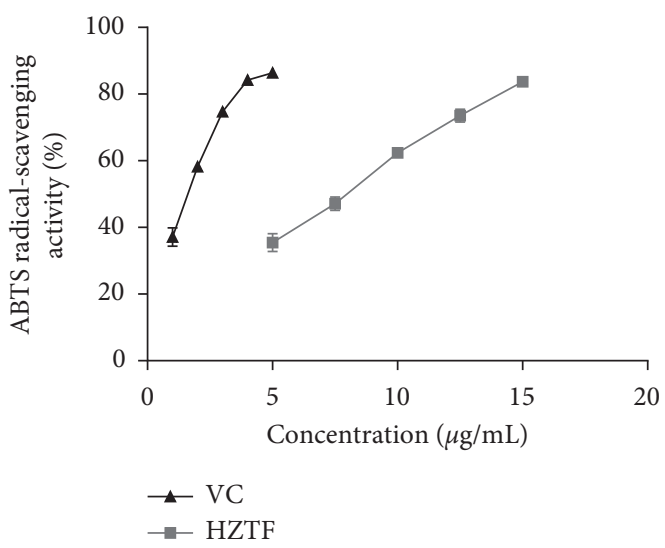

(c)

FIGURE 3: Lipid peroxidation inhibition ability. (a) DPPH free radical-scavenging activity and (b) $\mathrm{ABTS}^{+}$radical-scavenging capacity (c) of HZTF at different concentrations.

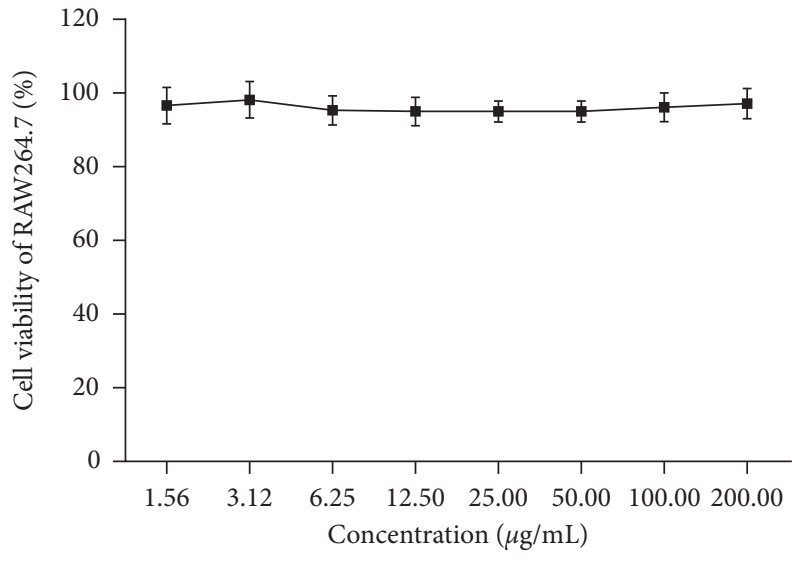

(a)

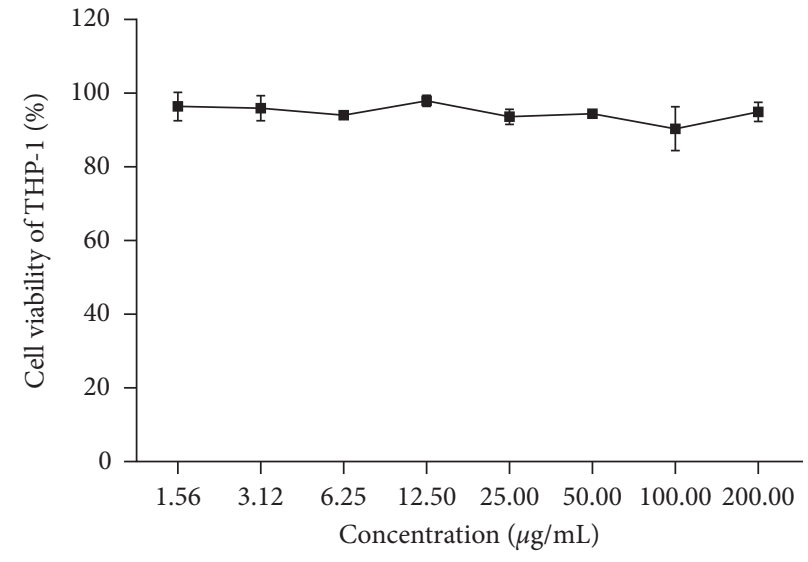

(b)

Figure 4: Cell viability of RAW264.7 (a) and THP-1 (b) cells treated with HZTF at different concentrations.

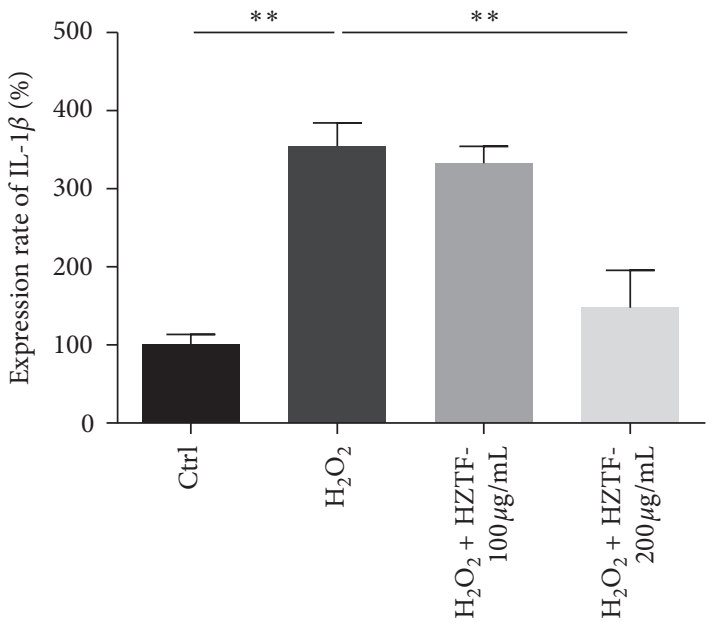

(a)

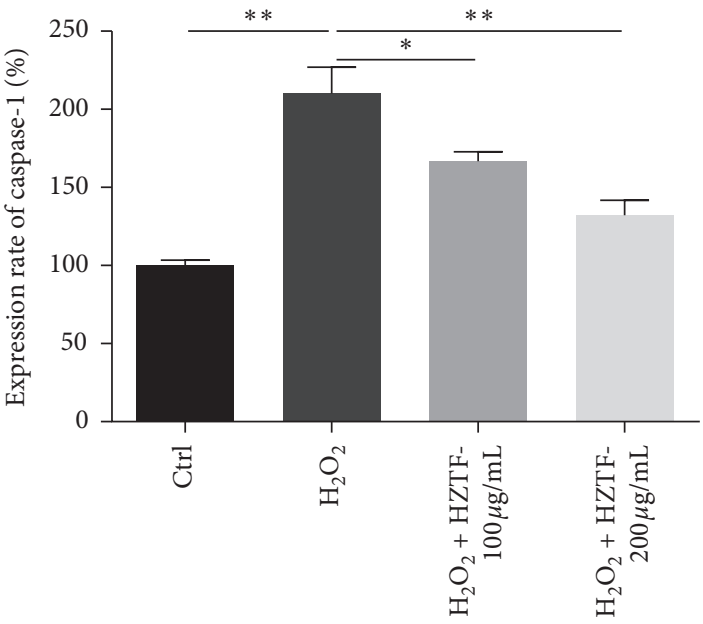

(b)

Figure 5: Continued. 


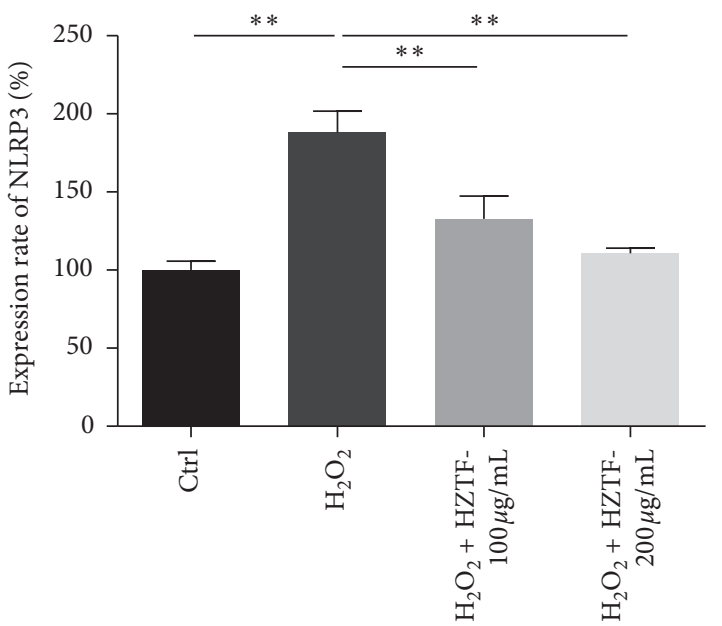

(c)

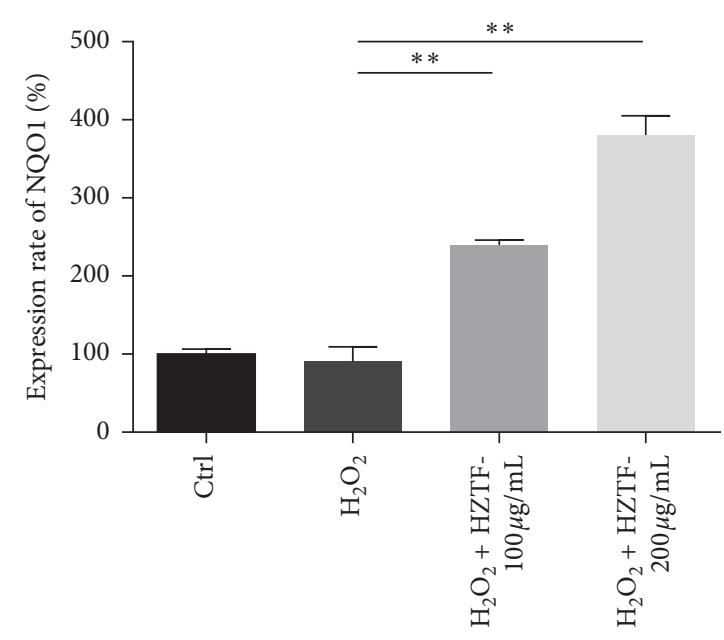

(d)

FIGURE 5: Effect of HZTF on IL-1 $\beta$ (a), caspase-1 (b), NLRP3 (c), and NQO1 (d) expression in $\mathrm{H}_{2} \mathrm{O}_{2-}$ stimulated RAW264.7 macrophages. The values are expressed as mean \pm SD. Comparisons are made with: control or HZTF $(100,200 \mu \mathrm{g} / \mathrm{ml})$ treated versus $\mathrm{H} 2 \mathrm{O} 2$-stimulated RAW264.7 macrophages. * means significantly different $(P<0.05)$ and ${ }^{* *}$ means very significantly different $(P<0.01)$.

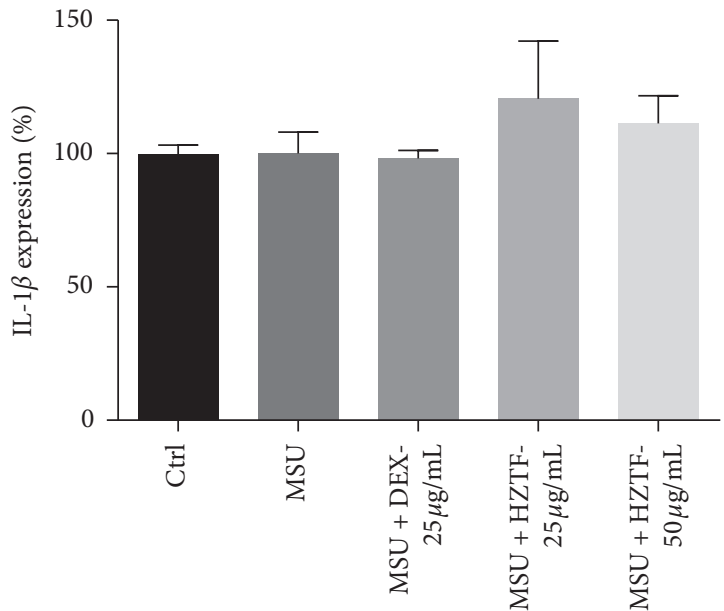

(a)

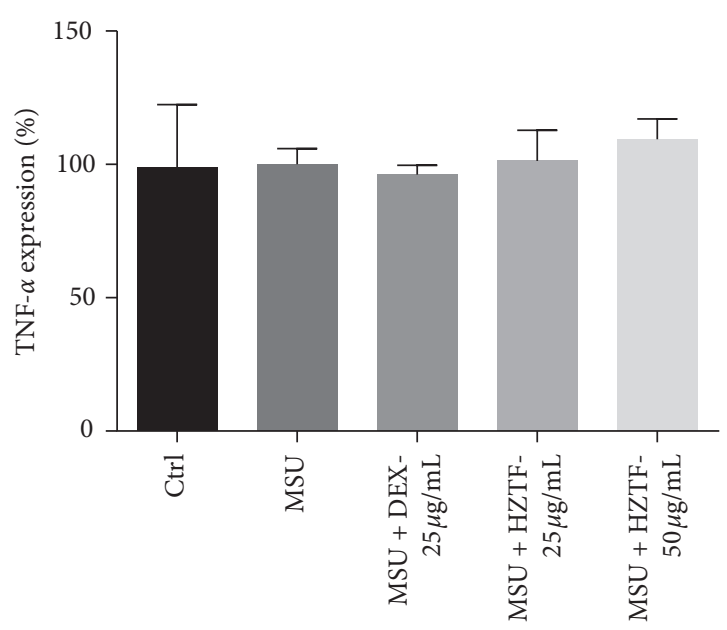

(c)

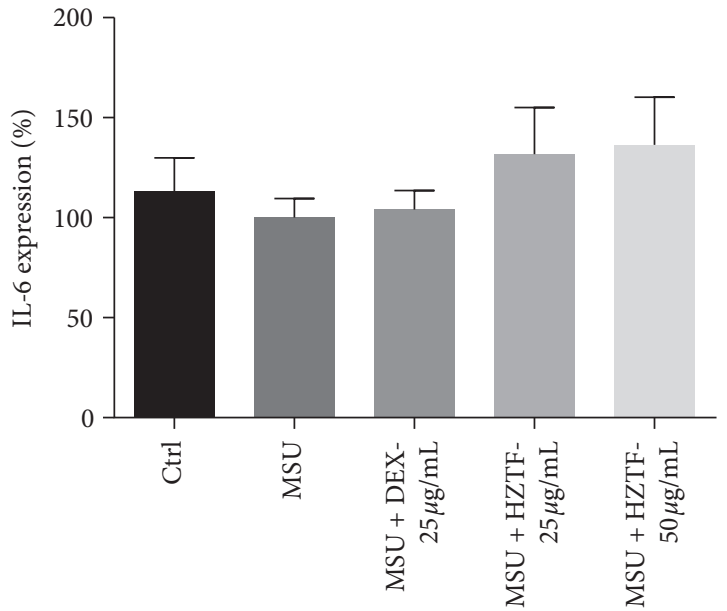

(b)

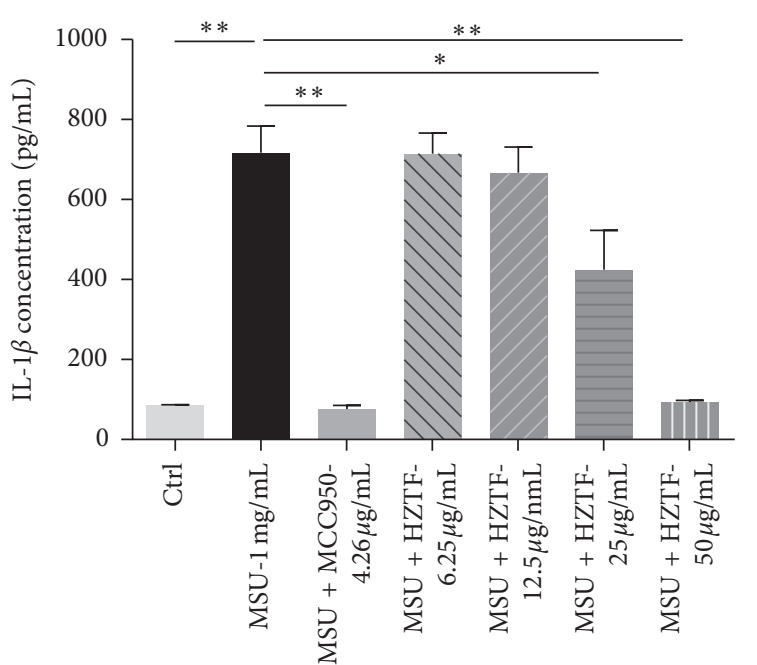

(d)

Figure 6: Continued. 


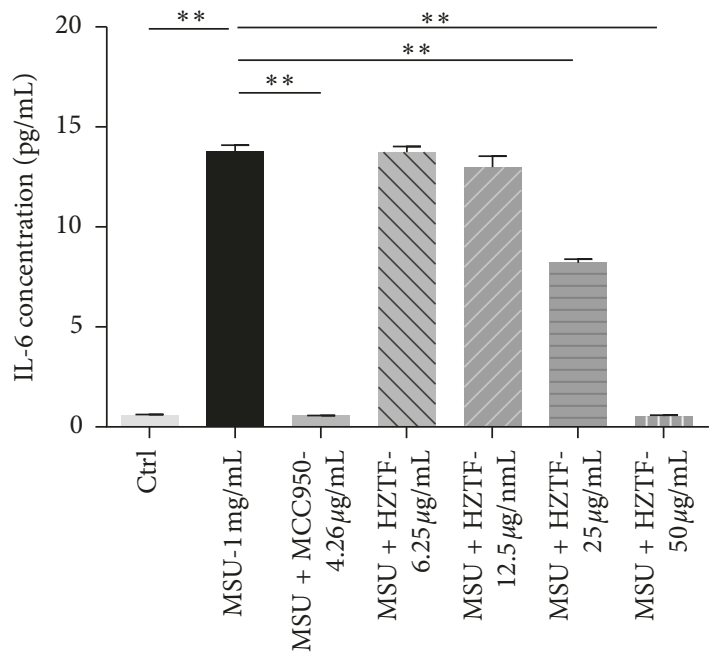

(e)

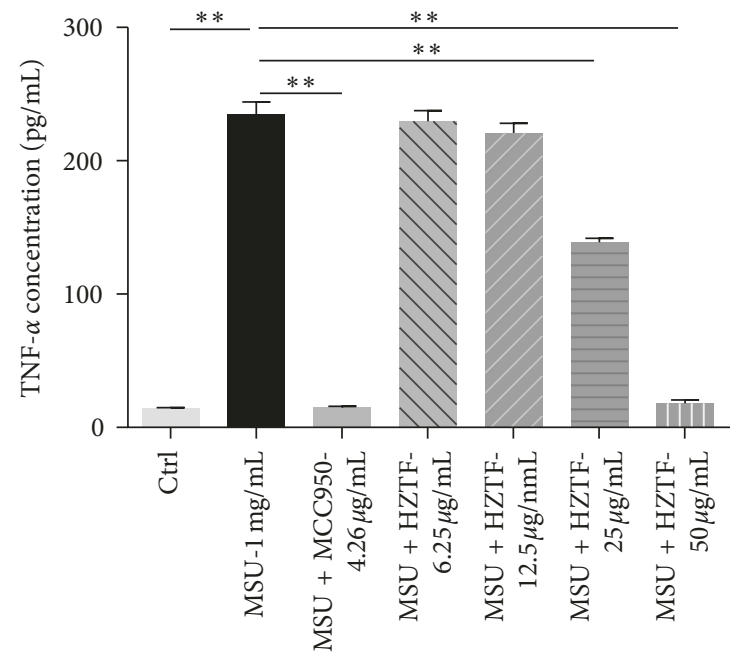

(f)

FIgURE 6: Effect of HZTF on IL-1 $\beta$ (a), IL-6 (b), and TNF- $\alpha$ (c) expression and IL-1 $\beta$ (d), IL-6 (e), and TNF- $\alpha$ (f) secretion in THP-1 stimulated by MSU. ${ }^{*}$ means significant different $(P<0.05)$ and ${ }^{* *}$ means very significant different $(P<0.01)$.
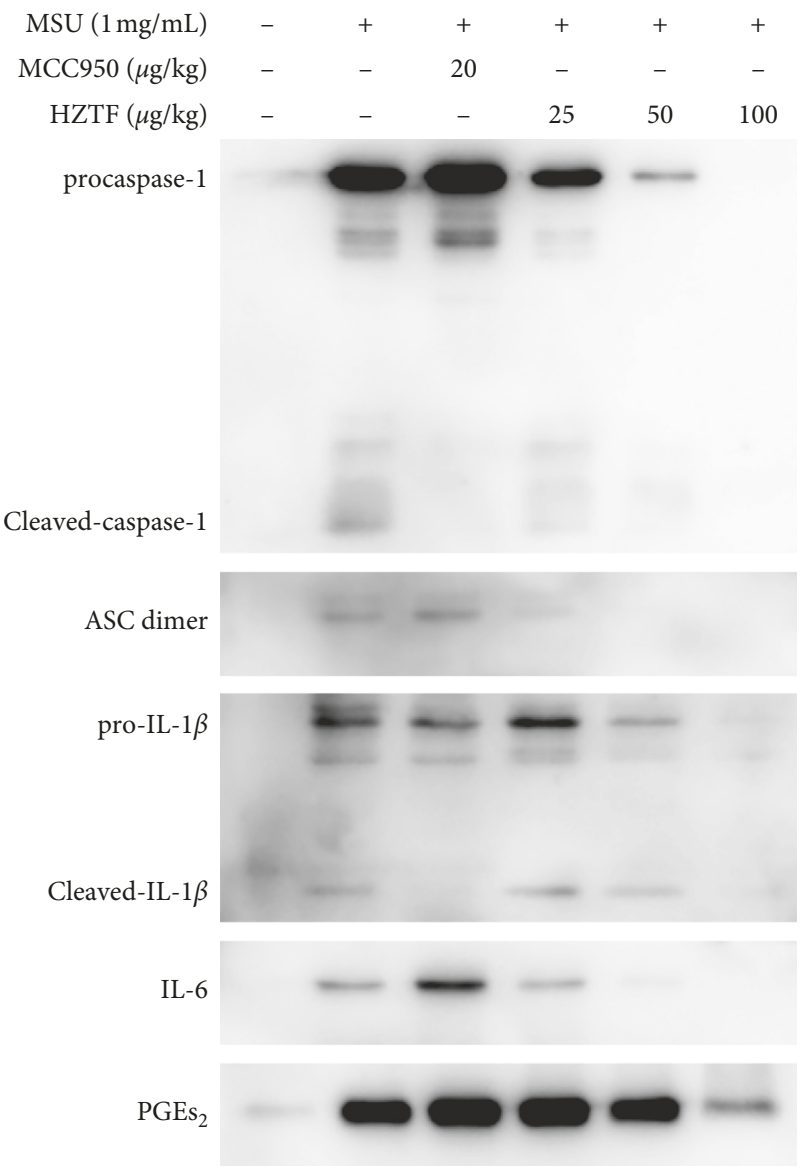

FIgURE 7: Effect of HZTF on procaspase-1, cleaved-caspase-1, proIL- $1 \beta$, cleaved-IL- $1 \beta$, ASC dimer, IL-6, and $\mathrm{PGE}_{2}$ protein expression secreted into culture medium.

species (ROS) oxidizing the biological membranes, which ROS reacts with membrane phospholipids, enzyme and membrane receptor-associated polyunsaturated side chain, and nucleic acid fatty acid and produces lipid peroxidation products such as malondialdehyde and 4-hydroxynonenal, and then the fluidity and permeability of cell membrane changed, resulting in the change of structure and function of cells $[37,38]$. The free radical-scavenging capacity of HZTF was measured using commercially available stable free radical DPPH. HZTF presented DPPH radical-scavenging capacity to some extent. It appears that antioxidant activity and reduction of DPPH both require the presence of a free $\mathrm{OH}$ group on the ellipticine ring [39], and if HZTF can reduce DPPH, it may suggest that HZTF contains compounds of similar structure. Based on the special chemical properties of formed free radicals, ABTS assay is frequently used to measure the radical-scavenging capacity of compounds [40]. The $\mathrm{ABTS}^{+}$radical elimination is a common antioxidant method as well as the DPPH' method to evaluate the antioxidant capacity of the chemical compound. Based on previous descriptions, Polygonum cuspidatum, Plantain, and Ligustrum, which were used to produce HZTF, can extract compounds with good antioxidant activity, and similar to HZTF, these compounds were also extracted from ethanol, which suggest that the main antioxidant compounds containing HZTF may be phenolic compounds too [41-43]. According to the results, the lipid peroxidation inhibition ability, DPPH radical-scavenging ability and $\mathrm{ABTS}^{+}$radical-scavenging capacity of HZTF were significantly weaker than VC $(P<0.05)$. VC is a strong antioxidant [44], but seldom used in acute gout treatment. HZTF has weaker antioxidant capacity than vitamin $\mathrm{C}$, but has a strong antigout capacity, so we believed that the antioxidant capacity of HZTF may not be the main mechanism of its antigout effect.

IL- $1 \beta$ is a proinflammatory cytokine that induces local and systemic inflammation aimed to eliminate foreign matters and microorganisms. However, exorbitant levels of inappropriate IL- $1 \beta$ production have been shown to be a key process in the etiology of the disease. In these conditions, 
DEPI

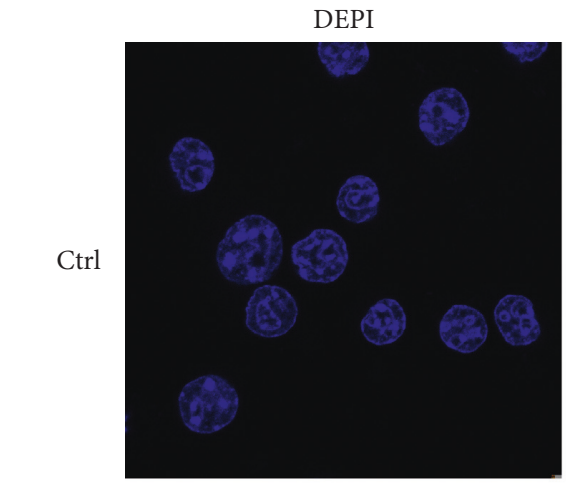

MSU
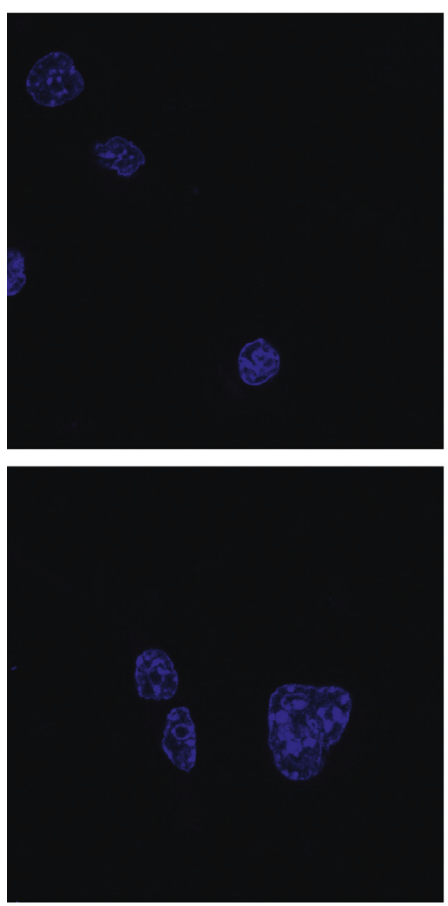

MCC950

HZTF

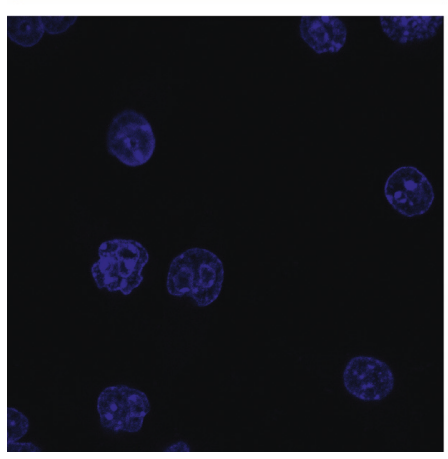

ASC
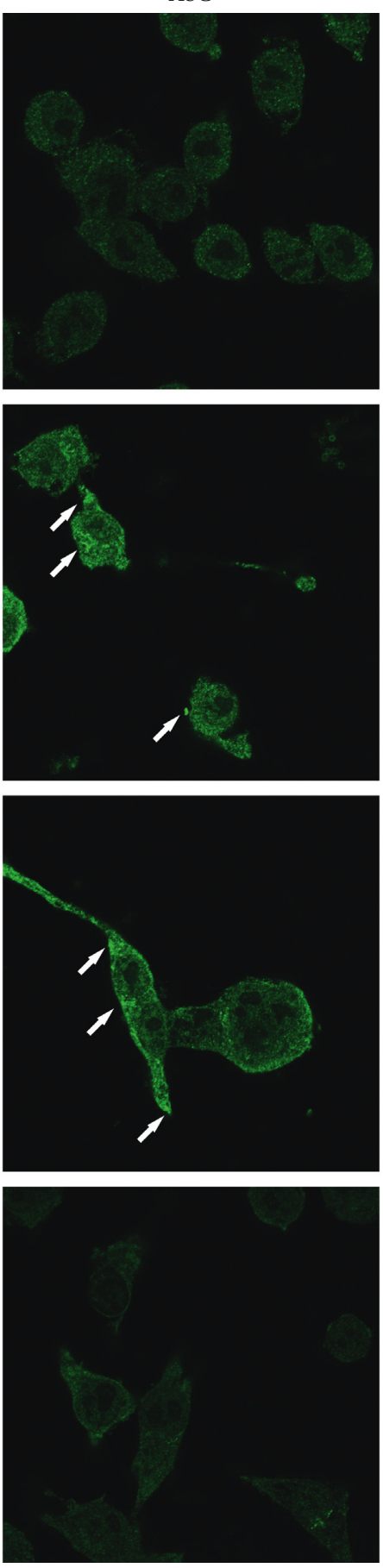

Merge
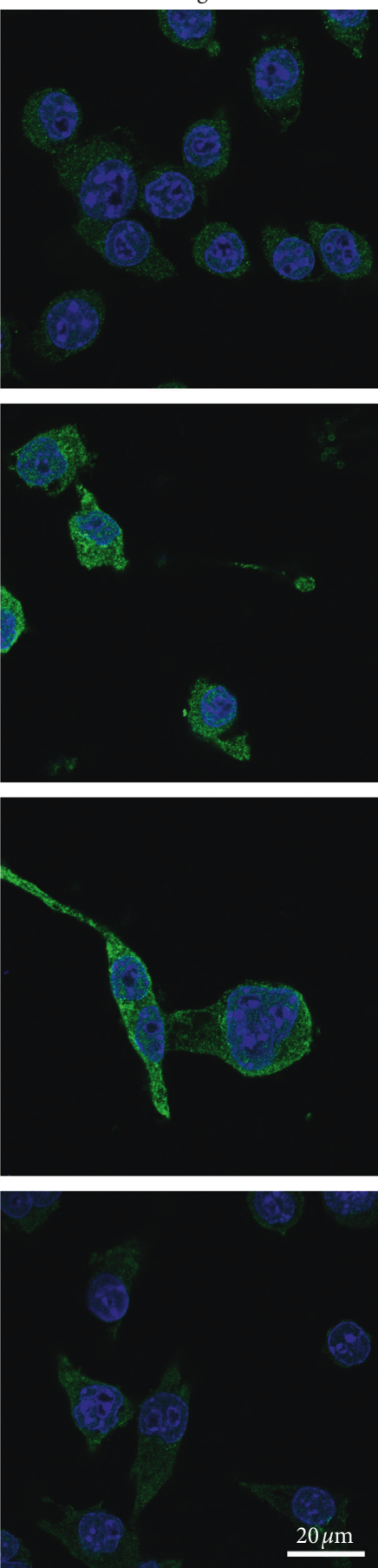

FIGURE 8: THP-1 cells were fixed, permeabilized, and stained for ASC (green), and the nuclei were stained with 4',6-diamidino-2phenylindole (DAPI; blue). The arrows indicate ASC speckles (Magnification, $\times 600$ ).

blocking IL- $1 \beta$ has proven very effective in clinical studies [45]. Caspase-1 and NLRP3 are closely linked to the pathogenesis of various metabolic diseases including gouty arthritis $[22,46]$. At the same time, the expression increase of IL- $1 \beta$, caspase- 1 , and NLRP3 is closely related to oxidative stress, and if we reduce the level of oxidative stress, we may suppress the expression of IL- $1 \beta$, caspase-1, and NLRP3 [47]. NQO1 is an important cellular antioxidant enzyme, and NQO1 is an Nrf2-regulated downstream enzyme, and the mRNA expression of NQO1 was observed after treatment. In the present study, we investigated whether HZTF has any regulatory effect on the gene expression of IL- $1 \beta$, caspase-1, NLRP3, and NQO1 in $\mathrm{H}_{2} \mathrm{O}_{2}$ stimulated RAW264.7 macrophages. Changes of IL-1 $\beta$, caspase-1, and NLRP3 expression in $\mathrm{H}_{2} \mathrm{O}_{2}$-induced RAW264.7 macrophages were compared with the control group without 
$\mathrm{H}_{2} \mathrm{O}_{2}$; the expression of IL- $1 \beta$, caspase-1, and NLRP3 in RAW264.7 macrophages was increased by treatment with $0.15 \% \mathrm{H}_{2} \mathrm{O}_{2}$ for $24 \mathrm{~h}$. Interestingly, the expression of IL- $1 \beta$, caspase-1, and NLRP3 in RAW264.7 macrophages treated with HZTF at 100.00 and $200.00 \mu \mathrm{g} / \mathrm{ml}$ were significantly reduced $(P<0.05)$. Furthermore, an obvious difference $(P<0.05)$ was found between the mRNA expression of NQO1 with or without HZTF $(100.00$ and $200.00 \mu \mathrm{g} / \mathrm{ml})$, the expression of NQO1 was increased with the increase of HZTF concentrations, which may indicate a dose-dependent relationship between NQO1 expression and HZTF concentrations. In conclusion, the results showed that $\mathrm{H}_{2} \mathrm{O}_{2}$ upregulated the expression of IL- $1 \beta$, caspase- 1 , and NLRP3, and downregulated the expression of NQO1. Treatments with HZTF inhibit the expression of IL-1 $\beta$, caspase-1, and NLRP3, and enhanced the expression of NQO1 in $\mathrm{H}_{2} \mathrm{O}_{2}$-induced RAW264.7 macrophages at the same time.

Development of the acute and chronic inflammatory responses known as gout and pseudogout are associated with the deposition of MSU crystals, in joints and periarticular tissues. MSU engage the caspase-1-activating NALP3 (also called cryopyrin) inflammasome, resulting in the production of active IL-1 $\beta$ [22]. Macrophages from mice deficient in components of the inflammasome such as ASC (RAW264.7 macrophages) are defective in crystal-induced IL- $1 \beta$ activation [48]. THP-1 cells were chosen for this experiment. According to the result, MSU crystals did not increase IL- $1 \beta$ mRNA expression, but increased IL- $1 \beta$ secretion, and HZTF also did not affect the mRNA expression of IL- $1 \beta$, IL- 6 , and TNF- $\alpha$, but significantly reduced the secretion of IL- $1 \beta$, IL- 6 , and TNF- $\alpha$ in THP-1 cells stimulated by MSU. In the western blotting test, we can know that HZTF inhibited procaspase-1, cleaved-caspase-1, pro-IL-1, cleaved-IL- $1 \beta$, and IL- 6 at the same time, suggesting that HZTF may inhibit not only the inflammasomes but also the NF- $\kappa$ b signaling. Also, in the western blotting result, HZTF showed inhibition effect in $\mathrm{PGE}_{2}$ secretion, which suggests that the mechanism of antigout effect of HZTF may be varied. At the same time, confocal microscopy analysis intuitively demonstrated the inhibitory effect of HZTF on ASC dimerization. These results suggested that HZTF may prevent gout by inhibiting NALP3 inflammasome as well as blocking the NF- $\kappa \mathrm{b}$ signaling pathway, which will increase the expression of IL- $1 \beta$, IL-6, and TNF- $\alpha$ [49].

Earlier reports indicated that four flavours of traditional Chinese medicinal materials which were used to produce HZTF contained several components, of which plantamajoside, Polygonum cuspidatum, specnuezhenide and resveratrol glucoside were found to be the main active constituent [2-11]. In present study, HPLC fingerprint analysis of HZTF showed distinct peaks of all four active constituents under $280 \mathrm{~nm}$ ultraviolet absorption.

\section{Conclusion}

The present study confirmed the antigout effects of HZTF in in vivo experiments and showed its reduction effects on the secretion of proinflammatory cytokines IL- $1 \beta$, IL-6, and
TNF- $\alpha$. At the same time, whether its antigout effect is related to its antioxidant capacity needs further study. This study provides a new promising therapeutic TCM for gout, and the precise mechanism of action is worth further to be investigated.

\section{Abbreviations}

TCM: Traditional chinese medicine

MSU: $\quad$ Monosodium urate

HZTF: Huzhentongfeng

VC: $\quad$ Ascorbic acid

DEX: Dexamethasone

DMEM: Dulbecco's modified eagle's medium

FBS: $\quad$ Fetal bovine serum

PBS: $\quad$ Phosphate-buffered saline

MTT: Methylthiazolyldiphenyl-tetrazolium bromide

PMA: Phorbol-12-myristate-13-acetat

DPPH: 1,1-Diphenyl-2-picryl-hydrazyl

ABTS: 3-Ethylbenzthiazoline-6-sulfonic acid

AAPH: $2,2^{\prime}$-Azobis (2-methylpropionamidine) dihydrochloride

Caspase- Cysteine-aspartic proteases-1

$1:$

NLRP3: NACHT, LRR, and PYD domains-containing protein 3

ASC: $\quad$ Apoptosis-associated speck-like protein containing

Caspase- Cysteinyl aspartate specific proteinase-1

1:

IL-1 $\beta$ : Interleukin- $1 \beta$

IL-6: $\quad$ Interleukin-6

TNF- $\alpha$ : Tumor necrosis factor- $\alpha$

$\mathrm{PGE}_{2}$ : $\quad$ Prostaglandin E2

NF- $\kappa$ B: Nuclear factor $-\kappa b$

NQO1: NADPH dehydrogenase, quinone-1

Nrf2: $\quad$ Nuclear factor (erythroid-derived 2)-like 2

HPLC: High-performance liquid chromatography

Elisa: Enzyme-linked immunosorbent assay

$\mathrm{IC}_{50}$ : Half maximal inhibitory concentration

H\&E: Hematoxylin and eosin

SD: $\quad$ Standard deviation

ANOVA: One-way analysis of variance

LPS: Lipopolysaccharide

PCR: $\quad$ Polymerase chain reaction

RT- Real-time quantitative polymerase chain qPCR: reaction.

\section{Data Availability}

The data used to support the findings of this study are available from the corresponding author upon request.

\section{Ethical Approval}

All studies were operated in accordance with the Institutional Animal Care Committee at the Experimental Animal Center of Henan Province and the China Council on Animal Care at Experimental Animal Center of Henan Province. 


\section{Conflicts of Interest}

The authors declare that they have no conflicts of interest.

\section{Authors' Contributions}

Z. C. W., Z. L. Z., and P. J. Z. investigated the effectiveness of HZTF as an antigouty inflammation medicine in MSU crystal-induced gouty rat in vivo and performed the cell-base experimental study; Q. Z., Z. Z., and J. P. D. performed the cell-base antioxidation experiment; K. Y. and H. F. performed the cell-free antioxidation experiment; and Y. F. W. and Z. P. W. were major contributors in designing the whole experimental and writing the manuscript. All authors read and approved the final manuscript.

\section{Acknowledgments}

This study was supported by GuangZhou (Jinan) Biomedical Research and Development Center by providing all the necessary facilities to carry out this work. This work was supported by the Guangdong Provincial Engineering Center of Topical Precise Drug Delivery System, the Science and Technology Projects of Guangdong Province (2016A04 0402033), and Guangzhou Major Program of the IndustryUniversity-Research Collaborative Innovation (20160404 6028). National Engineering Research Center for Modernization of Traditional Chinese Medicine-Sub Center of Artemisiae Argyi Folium, Tianhe District Science and Technology Plan Project-Key Technologies Project (2018BY003), Introduction of Leading Talents Program, Swan Project Innovation Team Plan of Huizhou City, and The Key Laboratory of Bioengineering Drugs of Guangdong Province of China funded for the reagent, animals, and instrument platform for all the experiments.

\section{References}

[1] A. K. So and F. Martinon, "Inflammation in gout: mechanisms and therapeutic targets," Nature Reviews: Rheumatology, vol. 13, no. 11, pp. 639-647, 2017.

[2] L. Sun, Z. D. He, R. M. Yang et al., "Hypolipidemic activity of total phenylpropanoid glycosides from Ligustrum robustum (Roxb.) blume and its mechanisms on AMPK pathway," Chinese Pharmacological Bulletin, vol. 33, no. 8, pp. 10731079, 2017.

[3] Q.-M. T. Ngo, H.-S. Lee, V. T. Nguyen, J. A. Kim, M. H. Woo, and B. S. Min, "Chemical constituents from the fruits of Ligustrum japonicum and their inhibitory effects on $\mathrm{T}$ cell activation," Phytochemistry, vol. 141, pp. 147-155, 2017.

[4] N. A. Campos, R. Swennen, and S. C. Carpentier, "The plantain proteome, a focus on allele specific proteins obtained from plantain fruits," Proteomics, vol. 18, no. 3-4, Article ID 1700227, 2018.

[5] L. R. Stuchlíková, L. Skálová, B. Szotáková et al., "Biotransformation of flubendazole and fenbendazole and their effects in the ribwort plantain (Plantago lanceolata)," Ecotoxicology and Environmental Safety, vol. 147, pp. 681-687, 2018.

[6] P. M. Fratellone, F. Tsimis, and G. Fratellone, "Apitherapy products for medicinal use," Journal of Nutrition \& Food Sciences, vol. 5, no. 6, pp. 461-462, 2015.
[7] D. Henatsch, F. Wesseling, K. W. Kross, and R. J. Stokroos, "Honey and beehive products in otorhinolaryngology: a narrative review," Clinical Otolaryngology, vol. 41, no. 5, pp. 519-531, 2016.

[8] J. Fu, S. Wu, M. Wang, Y. Tian, Z. Zhang, and R. Song, "Intestinal metabolism of polygonum cuspidatum in vitro and in vivo," Biomedical Chromatography, vol. 32, no. 6, Article ID e4190, 2018.

[9] W.-G. Ma, J. Wang, X.-W. Bu et al., "Effects of Polygonum cuspidatum on AMPK-FOXO3 $\alpha$ signaling pathway in a rat model of uric acid-induced renal damage," Chinese Journal of Integrative Medicine, vol. 25, no. 3, pp. 182-189, 2019.

[10] X. Guan, Y. Zhou, X. Liang, J. Xiao, L. He, and J. Li, "Effects of compounds found in Nidus vespae on the growth and cariogenic virulence factors of Streptococcus mutans," Microbiological Research, vol. 167, no. 2, pp. 61-68, 2012.

[11] M. Zhu, Y. Ling, Q. Qi, Y. Zhang, Y. Bao, and Y. Liu, "The immunomodulatory effects of Nidus vespae on human peripheral blood immune cells in vitro," Evidence-Based Complementary and Alternative Medicine, vol. 2015, Article ID 705308, 6 pages, 2015.

[12] W. Peng, R. Qin, X. Li, and H. Zhou, "Botany, phytochemistry, pharmacology, and potential application of Polygonum cuspidatum Sieb.et Zucc.: a review," Journal of Ethnopharmacology, vol. 148, no. 3, pp. 729-745, 2013.

[13] S.-Y. Zhou, Y.-M. Liu, Q.-Z. Zhang, Y.-W. Fu, and D.-J. Lin, "Evaluation of an antiparasitic compound extracted from Polygonum cuspidatum against Ichthyophthirius multifiliis in grass carp," Veterinary Parasitology, vol. 253, pp. 22-25, 2018.

[14] G. Peron, J. Uddin, M. Stocchero et al., "Studying the effects of natural extracts with metabolomics: a longitudinal study on the supplementation of healthy rats with Polygonum cuspidatum Sieb. et Zucc," Journal of Pharmaceutical and Biomedical Analysis, vol. 140, pp. 62-70, 2017.

[15] A. B. Samuelsen, "The traditional uses, chemical constituents and biological activities of Plantago major L. A review," Journal of Ethnopharmacology, vol. 71, no. 1-2, pp. 1-21, 2000.

[16] N. M. S. Palmeiro, C. E. Almeida, and P. C. Ghedini, "Oral subchronic toxicity of aqueous crude extract of Plantago australis leaves," Journal of Ethnopharmacology, vol. 88, no. 1, pp. 15-18, 2003.

[17] I. T. Stanisavljević, S. S. Stojičević, D. T. Veličković, M. L. Lazić, and V. B. Veljković, "Screening the antioxidant and antimicrobial properties of the extracts from plantain (Plantago Major L.) leaves," Separation Science \& Technology, vol. 43, no. 14, pp. 3652-3662, 2008.

[18] W. S. Suh, O. K. Kwon, T. H. Lee, L. Subedi, S. Y. Kim, and K. R. Lee, "Secoiridoid glycosides from the twigs of Ligustrum obtusifolium possess anti-inflammatory and neuroprotective effects," Chemical and Pharmaceutical Bulletin, vol. 66, no. 1, pp. 78-83, 2018.

[19] X. Pang, J.-Y. Zhao, H.-Y. Yu et al., "Secoiridoid analogues from the fruits of Ligustrum lucidum and their inhibitory activities against influenza A virus," Bioorganic \& Medicinal Chemistry Letters, vol. 28, no. 9, pp. 1516-1519, 2018.

[20] J.-B. He, Y.-M. Yan, X.-J. Ma et al., "Sesquiterpenoids and diarylheptanoids from Nidus vespae and their inhibitory effects on nitric oxide production," Chemistry \& Biodiversity, vol. 8, no. 12, pp. 2270-2276, 2011.

[21] B. P. Kamdem, M. Singh, M. A. Kumar, and A. Pal, "Acute and sub-chronic toxicity of HPLC fingerprinted extract of Conyza sumatrensis (Retz.) E. H. Walker in rodents," Journal of Ethnopharmacology, vol. 149, no. 3, pp. 833-837, 2013. 
[22] F. Martinon, V. Pétrilli, A. Mayor, A. Tardivel, and J. Tschopp, "Gout-associated uric acid crystals activate the NALP3 inflammasome," Nature, vol. 440, no. 7081, pp. 237-241, 2006.

[23] P. Costa, S. Gonçalves, P. Valentão, P. B. Andrade, and A. Romano, "Accumulation of phenolic compounds in in vitro cultures and wild plants of Lavandula viridis L'Hér and their antioxidant and anti-cholinesterase potential," Food and Chemical Toxicology, vol. 57, pp. 69-74, 2013.

[24] T. Ezure, T. Kanayama, and C. Nishino, "Action of the novel antioxidants 4GBE43 and 2BBE43 against lipid peroxidation 1," Biochemical Pharmacology, vol. 62, no. 3, pp. 335-340, 2001.

[25] Y. Kimuya, M. Kubo, T. Tani, S. Arichi, and H. Okuda, "Studies on Scutellariae Radix. IV. Effects on lipid peroxidation in rat liver," Chemical and Pharmaceutical Bulletin, vol. 29, no. 9, pp. 2610-2617, 1981.

[26] W. Luo, M. Zhao, B. Yang, G. Shen, and G. Rao, "Identification of bioactive compounds in Phyllenthus emblica L. fruit and their free radical scavenging activities," Food Chemistry, vol. 114, no. 2, pp. 499-504, 2009.

[27] J. Emerit, J. Fechner, A. Galli, J. P. Clavel, and F. Congy, "Free radicals derived from oxygen and lipid peroxidation. Role in cellular biology and physiopathology," La Presse médicale, vol. 15, no. 16, pp. 751-754, 1986.

[28] T. Yamaguchi, H. Takamura, T. Matoba, and J. Terao, "HPLC method for evaluation of the free radical-scavenging activity of foods by using 1,1-diphenyl-2-picrylhydrazyl," Bioscience Biotechnology \& Biochemistry, vol. 62, no. 6, pp. 1201-1204, 1998.

[29] C. A. Rice-Evans, N. J. Miller, and G. Paganga, "Structureantioxidant activity relationships of flavonoids and phenolic acids," Free Radical Biology and Medicine, vol. 20, no. 7, pp. 933-956, 1996.

[30] R. V. D. Werf, C. Marcic, A. Khalil, S. Sigrist, and E. Marchioni, "ABTS radical scavenging capacity in green and roasted coffee extracts," LWT-Food Science and Technology, vol. 58, no. 1, pp. 77-85, 2014.

[31] R. Re, N. Pellegrini, A. Proteggente, A. Pannala, M. Yang, and C. Rice-Evans, "Antioxidant activity applying an improved ABTS radical cation decolorization assay," Free Radical Biology and Medicine, vol. 26, no. 9-10, pp. 1231-1237, 1999.

[32] M. A. Martillo, L. Nazzal, and D. B. Crittenden, "The crystallization of monosodium urate," Current Rheumatology Reports, vol. 16, no. 2, p. 400, 2014.

[33] S. Schlee, L. C. Bollheimer, T. Bertsch, C. C. Sieber, and P. Härle, "Crystal arthritides-gout and calcium pyrophosphate arthritis," Zeitschrift für Gerontologie und Geriatrie, vol. 51, no. 6, pp. 703-710, 2018.

[34] F. Apel, A. Zychlinsky, and E. F. Kenny, "The role of neutrophil extracellular traps in rheumatic diseases," Nature Reviews: Rheumatology, vol. 14, no. 8, pp. 467-475, 2018.

[35] M. Napagoda, J. Gerstmeier, H. Butschek et al., "Lipophilic extracts of Leucas zeylanica, a multi-purpose medicinal plant in the tropics, inhibit key enzymes involved in inflammation and gout," Journal of Ethnopharmacology, vol. 224, pp. 474481, 2018.

[36] L. Li, M. Teng, Y. Liu et al., "Anti-gouty arthritis and antihyperuricemia Effects of sunflower head Extract in gouty and hyperuricemia animal models," BioMed Research International, vol. 2017, Article ID 5852076, 9 pages, 2017.

[37] L. J. Marnett, "Lipid peroxidation-DNA damage by malondialdehyde," Mutation Research/Fundamental and Molecular Mechanisms of Mutagenesis, vol. 424, no. 1-2, pp. 83-95, 1999.
[38] M. Trevisan, "Correlates of markers of oxidative status in the general population," American Journal of Epidemiology, vol. 154, no. 4, pp. 348-356, 2001.

[39] C. Rousseau-Richard, C. Auclair, C. Richard, and R. Martin, "Free radical scavenging and cytotoxic properties in the ellipticine series," Free Radical Biology \& Medicine, vol. 8, no. 3, pp. 223-230, 1990.

[40] D. Huang, B. Ou, and R. L. Prior, "The chemistry behind antioxidant capacity assays," Journal of Agricultural and Food Chemistry, vol. 53, no. 6, pp. 1841-1856, 2005.

[41] C.-Y. Hsu, Y.-P. Chan, and J. Chang, "Antioxidant activity of extract from Polygonum cuspidatum," Biological Research, vol. 40, no. 1, pp. 13-21, 2007.

[42] L. Yu, J.-X. Ren, H.-M. Nan, and B.-F. Liu, "Identification of antibacterial and antioxidant constituents of the essential oils of Cynanchum chinense and Ligustrum compactum," Natural Product Research, vol. 29, no. 18, pp. 1779-1782, 2015.

[43] C. G. Pereira, L. Custódio, M. J. Rodrigues et al., "Profiling of antioxidant potential and phytoconstituents of Plantago coronopus," Brazilian Journal of Biology, vol. 77, no. 3, pp. 632-641, 2016.

[44] J. Enko and A. Gliszczyńska-Swigło, "Influence of the interactions between tea (Camellia sinensis) extracts and ascorbic acid on their antioxidant activity: analysis with interaction indexes and isobolograms," Food Additives \& Contaminants, vol. 32, no. 8, pp. 1234-1242, 2015.

[45] A. Mistry, S. Savic, and J. C. H. van der Hilst, "Interleukin-1 blockade: an update on emerging indications," Biodrugs, vol. 31, no. 3, pp. 207-221, 2017.

[46] H. E. Lee, G. Yang, N. D. Kim et al., “Targeting ASC in NLRP3 inflammasome by caffeic acid phenethyl ester: a novel strategy to treat acute gout," Scientific Reports, vol. 6, no. 1, Article ID 38622, 2016.

[47] P. Dinesh and M. K. Rasool, "Berberine, an isoquinoline alkaloid suppresses TXNIP mediated NLRP3 inflammasome activation in MSU crystal stimulated RAW 264.7 macrophages through the upregulation of Nrf2 transcription factor and alleviates MSU crystal induced inflammation in rats," International Immunopharmacology, vol. 44, pp. 26-37, 2017.

[48] W.-T. He, H. Wan, L. Hu et al., "Gasdermin D is an executor of pyroptosis and required for interleukin- $1 \beta$ secretion," Cell Research, vol. 25, no. 12, pp. 1285-1298, 2015.

[49] K.-K. Jia, H. Ding, H.-W. Yu, T.-J. Dong, Y. Pan, and L.-D. Kong, "Huanglian-wendan decoction inhibits NF-B/ NLRP3 inflammasome activation in liver and brain of rats exposed to chronic unpredictable mild stress," Mediators of Inflammation, vol. 2018, Article ID 3093516, 15 pages, 2018. 


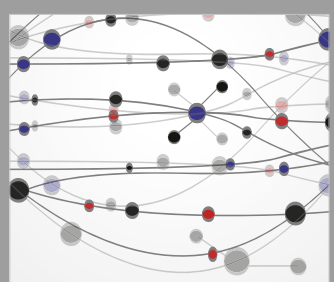

The Scientific World Journal
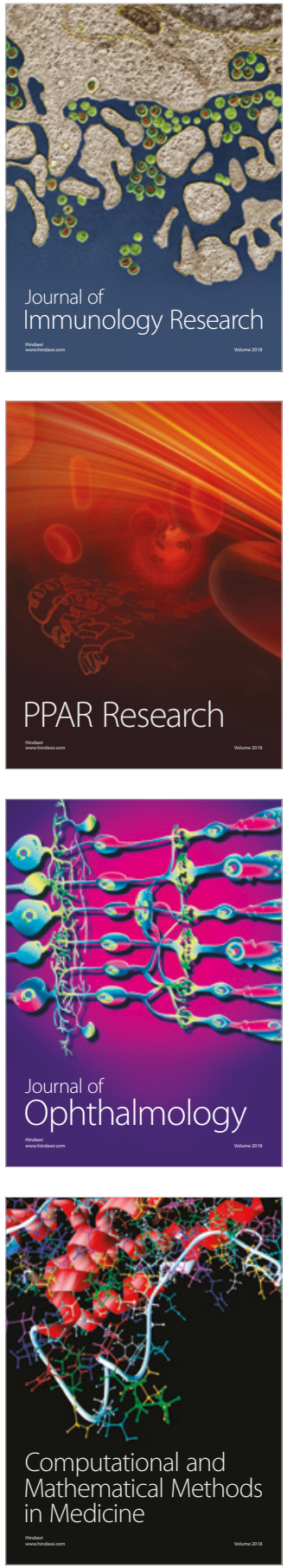

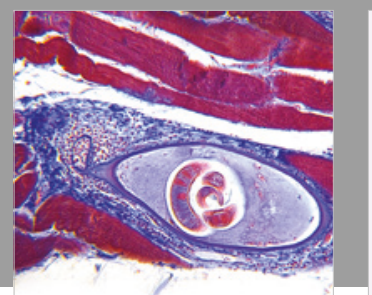

Gastroenterology Research and Practice

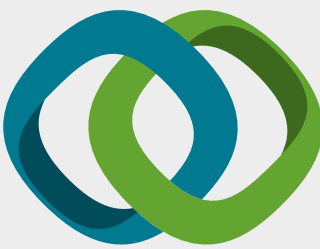

\section{Hindawi}

Submit your manuscripts at

www.hindawi.com
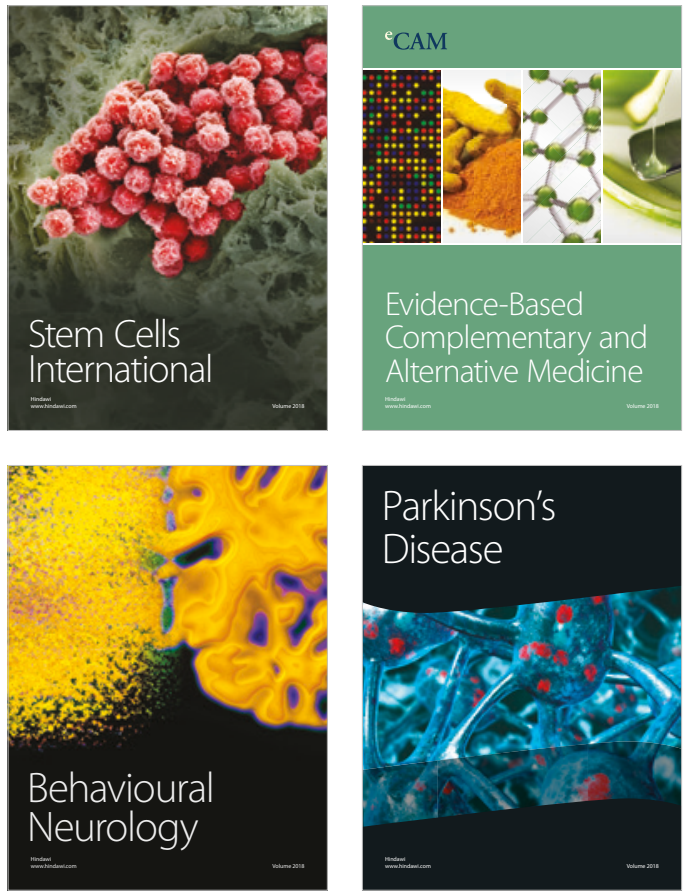

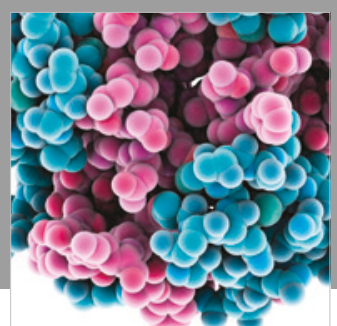

ournal of

Diabetes Research

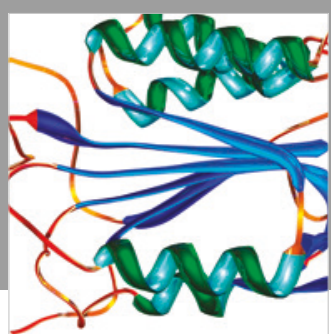

Disease Markers
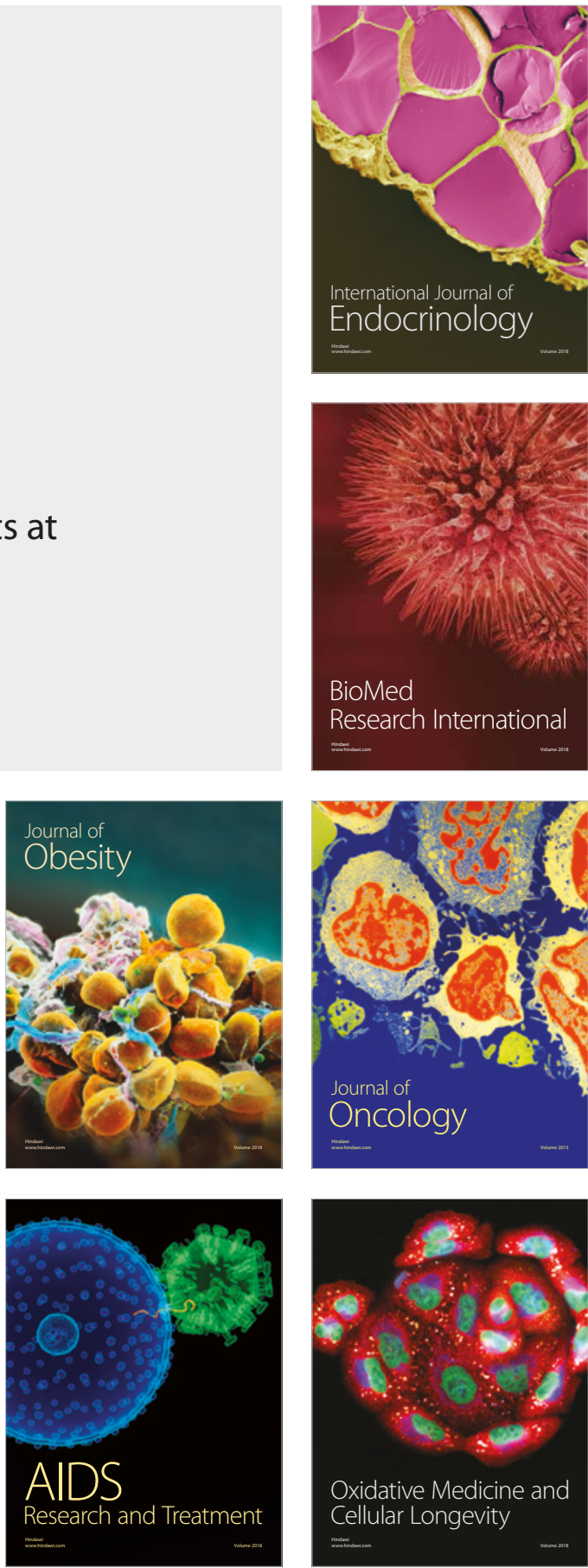